\title{
Classifying and Applying Rational Knots and Rational Tangles
}

\author{
Louis H. Kauffman and Sofia Lambropoulou
}

\begin{abstract}
In this survey paper we sketch new combinatorial proofs of the classification of rational tangles and of unoriented and oriented rational knots, using the classification of alternating knots and the calculus of continued fractions. We continue with the classification of achiral and strongly invertible rational links, and we conclude with a description of the relationships among tangles, rational knots and DNA recombination.
\end{abstract}

\section{Introduction}

Rational knots and links, also known in the literature as four-plats, Viergeflechte and 2-bridge knots, are a class of alternating links of one or two unknotted components and they are the easiest knots to make (also for Nature!). The first twenty five knots, except for $8_{5}$, are rational. Furthermore all knots up to ten crossings are either rational or are obtained from rational knots by certain simple operations. Rational knots give rise to the lens spaces through the theory of branched coverings. A rational tangle is the result of consecutive twists on neighbouring endpoints of two trivial arcs, see Definition 2.1. Rational knots are obtained by taking numerator closures of rational tangles (see Figure 5), which form a basis for their classification. Rational knots and rational tangles are of fundamental importance in the study of DNA recombination. Rational knots and links were first considered in $[\mathbf{2 8}]$ and $[\mathbf{1}]$. Treatments of various aspects of rational knots and rational tangles can be found in $[\mathbf{5}],[\mathbf{3 4}],[\mathbf{4}],[\mathbf{3 0}],[\mathbf{1 4}],[\mathbf{1 7}],[\mathbf{2 0}],[\mathbf{2 3}]$. See also $[\mathbf{2}]$ for a good discussion on classical relationships of rational tangles, covering spaces and surgery. A rational tangle is associated in a canonical manner with a unique, reduced rational number or $\infty$, called the fraction of the tangle. Rational tangles are classified by their fractions by means of the following theorem:

TheOREm 1.1 (Conway, 1970). Two rational tangles are isotopic if and only if they have the same fraction.

1991 Mathematics Subject Classification. 57M25, 57M27.

Key words and phrases. rational knots and links, 2-tangles, rational tangles, continued fractions, tangle fraction, coloring, chirality, invertibility, DNA recombination. 
John H. Conway [5] introduced the notion of tangle and defined the fraction of a rational tangle using the continued fraction form of the tangle and the Alexander polynomial of knots. Conway was the first to observe the extraordinary interplay between the elementary number theory of fractions and continued fractions, and the topology of rational tangles and rational knots and links.

Proofs of Theorem 1.1 can be found in [22], [4] p.196, [13] and [15]. The first two proofs invoke the classification of rational knots and the theory of branched covering spaces. The proof by Goldman and Kauffman [13] is the first combinatorial proof of this theorem. In [15] the proof is combinatorial and the topological invariance of the fraction of a rational tangle is proved via flyping and also via coloring.

More than one rational tangle can yield the same or isotopic rational knots and the equivalence relation between the rational tangles is reflected into an arithmetic equivalence of their corresponding fractions. This is marked by a theorem due originally to Schubert [33] and reformulated by Conway [5] in terms of rational tangles.

THEOREM 1.2 (Schubert, 1956). Suppose that rational tangles with fractions $\frac{p}{q}$ and $\frac{p^{\prime}}{q^{\prime}}$ are given ( $p$ and $q$ are relatively prime. Similarly for $p^{\prime}$ and $q^{\prime}$.) If $K\left(\frac{p}{q}\right)$ and $K\left(\frac{p^{\prime}}{q^{\prime}}\right)$ denote the corresponding rational knots obtained by taking numerator closures of these tangles, then $K\left(\frac{p}{q}\right)$ and $K\left(\frac{p^{\prime}}{q^{\prime}}\right)$ are topologically equivalent if and only if

1. $p=p^{\prime}$ and

2. either $q \equiv q^{\prime}(\bmod p)$ or $q q^{\prime} \equiv 1(\bmod p)$.

This classic theorem [33] has hitherto been proved by using the observation of Seifert [31] that the 2-fold branched covering spaces of $S^{3}$ along $K\left(\frac{p}{q}\right)$ and $K\left(\frac{p^{\prime}}{q^{\prime}}\right)$ are lens spaces, and invoking the results of Reidemeister [29] on the classification of lens spaces. Another proof using covering spaces has been given by Burde in [3]. Schubert also extended this theorem to the case of oriented rational knots and links described as 2-bridge links:

THEOREM 1.3 (Schubert, 1956). Suppose that orientation-compatible rational tangles with fractions $\frac{p}{q}$ and $\frac{p^{\prime}}{q^{\prime}}$ are given with $q$ and $q^{\prime}$ odd. ( $p$ and $q$ are relatively prime. Similarly for $p^{\prime}$ and $q^{\prime}$.) If $K\left(\frac{p}{q}\right)$ and $K\left(\frac{p^{\prime}}{q^{\prime}}\right)$ denote the corresponding rational knots obtained by taking numerator closures of these tangles, then $K\left(\frac{p}{q}\right)$ and $K\left(\frac{p^{\prime}}{q^{\prime}}\right)$ are topologically equivalent if and only if

1. $p=p^{\prime}$ and

2. either $q \equiv q^{\prime}(\bmod 2 p)$ or $q q^{\prime} \equiv 1(\bmod 2 p)$.

In [16] we give the first combinatorial proofs of Theorem 1.2 and Theorem 1.3. Our methods for proving these results are in fact methods for understanding these knots at the diagrammatic level. We have located the essential points in the proof of the classification of rational knots in the direct combinatorics related to the question: Which rational tangles will close to form this specific knot or link diagram? By looking at the theorems in this way, we obtain a path to the results that can be understood without extensive background in three-dimensional topology. This allows us to explain deep results in an elementary fashion. 
In this paper we sketch the proofs in $[\mathbf{1 5}]$ and $[\mathbf{1 6}]$ of the above three theorems and we give the key examples that are behind all of our proofs. In order to compose elementary proofs, we have relied on a deep result in topology - namely the solution by Menasco and Thistlethwaite [21] of the Tait Conjecture [37] concerning the classification of alternating knots. The Tait Conjecture is easily stated and understood. Hence it provides an ideal tool for our exploration. The present paper constitutes an introduction to our work in this domain and it will be of interest to biologists and mathematicians. We intend it to be accessible to anyone who is beginning to learn knot theory and its relationship with molecular biology. In most cases the detailed proofs are not given here, but can be found in our research papers $[\mathbf{1 5}],[\mathbf{1 6}]$. We also give some applications of Theorems 1.2 and 1.3 using our methods.

The paper is organized as follows. In Section 2 we introduce 2-tangles, their isotopies and operations, and we state the Tait Conjecture. In Section 3 we introduce the rational tangles as a special class of 2-tangles, and we show how to extract continued fraction expressions for rational tangles. The section concludes with a proof that rational tangles are alternating, which implies a unique canonical form for rational tangles. In Section 4 we recall facts about finite continued fractions with numerators equal to 1 and give a unique canonical form for continued fractions. Then we associate a continued fraction to a rational tangle. The arithmetic value of this continued fraction is called the fraction of the tangle. We then present the classification of rational tangles (Theorem 1.1) in terms of their fractions by unravelling the relationship between the topological and arithmetical operations on rational tangles and rational numbers. At the end of Section 4 we give an alternate definition of the fraction of a rational tangle using the method of integral coloring.

In Section 5 we give a sketch of our proof of Theorem 1.2 of the classification of unoriented rational knots by means of a direct combinatorial and arithmetical analysis of rational knot diagrams, using the Tait Conjecture and the classification of rational tangles. In Section 6 we discuss chirality of knots and give a classification of the achiral rational knots and links as numerator closures of even palindromic rational tangles in continued fraction form (Theorem 6.1). In Section 7 we give our interpretation of the statement of Theorem 1.3 and we sketch our proof of the classification of oriented rational knots, using the methods we developed in the unoriented case, and examining the connectivity patterns of oriented rational knots (Theorem 7.1).

In Section 8 we point out that all oriented rational knots and links are invertible. This section gives a classification of the strongly invertible rational links (reverse the orientation of one component) as closures of odd palindromic oriented rational tangles in continued fraction form (Theorem 8.1). The paper ends with an introduction to the application of these methods to DNA recombination. Section 9 outlines the tangle model of DNA recombination (see [35]) as an application of Theorem 1.2, and it gives a bound on the needed number of DNA recombination experiments for solving certain tangle equations (Theorem 9.1). 


\section{Rational Tangles and their Operations}

Throughout this paper we will be working with tangles. The theory of tangles was discovered by John Conway [5] in his work on enumerating and classifying knots. An $(m, n)$-tangle is an embedding of a finite collection of arcs (homeomorphic to the interval $[0,1]$ ) and circles into the three-dimensional Euclidean space, such that the endpoints of the arcs go to a specific set of $m+n$ points on the surface of a ball $B^{3}$ standardly embedded in $S^{3}$, so that the $m$ points lie on the upper hemisphere and the $n$ points on the lower hemisphere with respect to the height function, and so that the circles and the interiors of the arcs are embedded in the interior of this ball. An $(n, n)$-tangle will be abbreviated to $n$-tangle. Knots and links are 0-tangles, and braids on $n$ strands are the most well-known class of $n$-tangles. The left-hand side of Figure 1 illustrates a 2-tangle. Finally, an $(m, n)$ tangle is oriented if we assign orientations to each arc and each circle. By definition, the total number of free strands, $m+n$, is required to be even, and without loss of generality the $m+n$ endpoints of a tangle can be arranged on a great circle on the sphere or in a box, which may also be omitted. One can then define a diagram of an $(m, n)$-tangle to be a regular projection of the tangle on the plane of this great circle. As we shall see below, the class of 2-tangles is of particular interest.
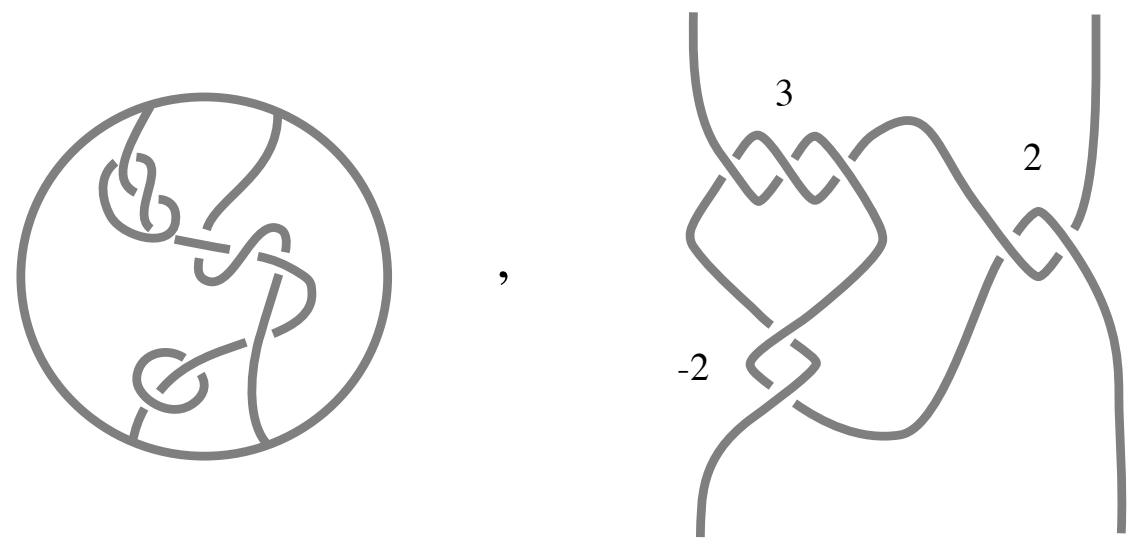

Figure 1. A 2-tangle and a rational tangle

We will soon concentrate on a special class of 2-tangles, the rational tangles. The simplest possible rational tangles comprise two unlinked arcs either horizontal or vertical. These are the trivial tangles, denoted [0] and $[\infty]$ tangles respectively, see Figure 2.

DEFINITION 2.1. Let $t$ be a pair of unoriented arcs properly embedded in a 3 -ball $B$. A 2-tangle is rational if there exists an orientation preserving homeomorphism of pairs:

$$
g:(B, t) \longrightarrow\left(D^{2} \times I,\{x, y\} \times I\right) \quad(\text { a trivial tangle }) .
$$

Definition 2.1 is equivalent to saying that rational tangles can be obtained by applying a finite number of consecutive twists of neighbouring endpoints to the 
elementary tangles [0] or $[\infty]$. In one direction, the act of untwisting a horizontal twist (e.g. the outer of the twists labeled 2 in Figure 1) can be expressed by such a homeomorphism of pairs. To see the equivalence of Definition 1 , let $S^{2}$ denote the two-dimensional sphere, which is the boundary of the 3-ball $B$ and let $p$ denote four specified points in $S^{2}$. Let further $h:\left(S^{2}, p\right) \longrightarrow\left(S^{2}, p\right)$ be a self-homeomorphism of $S^{2}$ with the four points. This extends to a self-homeomorphism $\bar{h}$ of the 3 -ball $B$ (see [30], page 10). Further, let $a$ denote the two straight arcs $\{x, y\} \times I$ joining pairs of the fours point of the boundary of $B$. Consider now $\bar{h}(a)$. We call this the tangle induced by $h$. We note that up to isotopy (see definition below) $h$ is a composition of braidings of pairs of points in $S^{2}$ (see [26], pages 61 to 65). Each such braiding induces a twist in the corresponding tangle. So, if $h$ is a composition of braidings of pairs of points, then the extension $\bar{h}$ is a composition of twists of neighbouring end arcs. Thus $\bar{h}(a)$ is a rational tangle and every rational tangle can be obtained this way. We shall use this equivalence as the characterizing property of rational tangles. Of course, each twisting operation changes the isotopy class of the tangle to which it is applied. Examples of rational tangles are illustrated in the right-hand side of Figure 1 as well as in Figures 7 and 10 below.

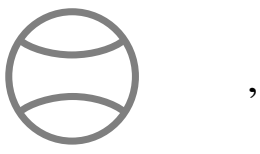

[0]

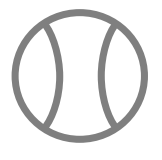

$[\infty]$

Figure 2. The trivial tangles $[0]$ and $[\infty]$

We are interested in studying tangles up to an equivalence relation called isotopy. Two $(m, n)$-tangles, $T, S$, in $B^{3}$ are said to be isotopic, denoted by $T \sim S$, if they have identical configurations of their $m+n$ endpoints in $S^{2}=\partial B^{3}$, and if there is an ambient isotopy of $\left(B^{3}, T\right)$ to $\left(B^{3}, S\right)$ that is the identity on the boundary $\left(S^{2}, \partial T\right)=\left(S^{2}, \partial S\right)$. An ambient isotopy can be imagined as a continuous deformation of $B^{3}$ fixing the $m+n$ endpoints on the boundary sphere, and bringing one tangle to the other without causing any self-intersections. Equivalently, there is an orientation-preserving self-homeomorphism $h:\left(B^{3}, T\right) \longrightarrow\left(B^{3}, S\right)$ that is the identity map on the boundary. Isotopic tangles are said to be in the same topological class.

In terms of diagrams, Reidemeister $[\mathbf{2 7}]$ proved that the local moves on diagrams illustrated in Figure 3 capture combinatorially the notion of ambient isotopy of knots, links and tangles in three-dimensional space. That is, if two diagrams represent knots, links or tangles that are isotopic, then the one diagram can be obtained from the other by a sequence of Reidemeister moves. In the case of tangles the endpoints of the tangle remain fixed and all the moves occur inside the tangle box.

Two oriented $(m, n)$-tangles are are said to be oriented isotopic if there is an isotopy between them that preserves the orientations of the corresponding arcs and the corresponding circles. The diagrams of two oriented isotopic tangles differ by a sequence of oriented Reidemeister moves, i.e. Reidemeister moves with orientations 
on the little arcs that remain consistent during the moves. From now on we will be thinking in terms of tangle diagrams. Also, we will be referring to both knots and links whenever we say 'knots'.

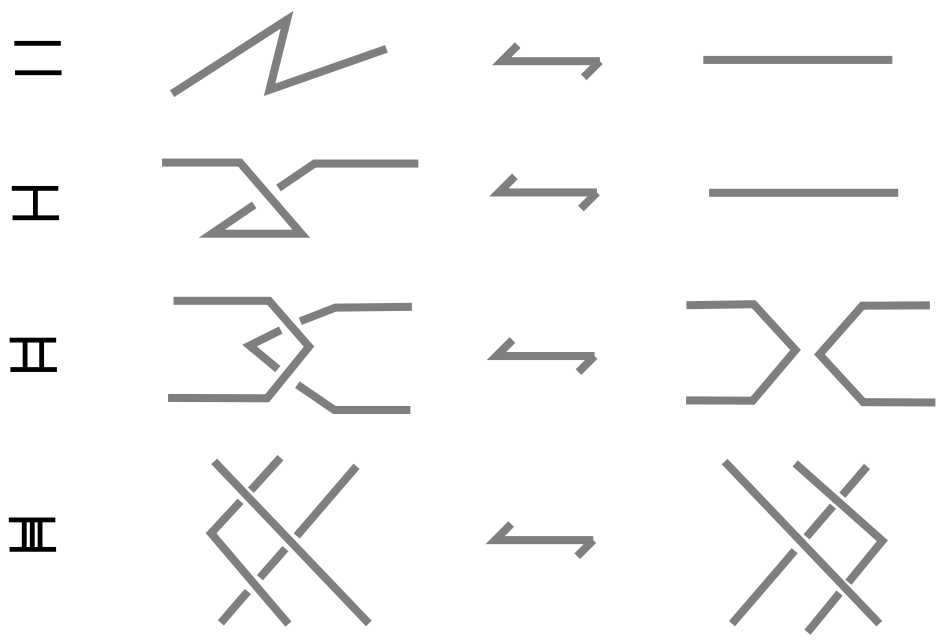

Figure 3. The Reidemeister moves

Let $T_{(m, n)}$ denote the set of all $(m, n)$ tangles. Among all tangles, the class $T_{(2,2)}$ of 2-tangles is particularly interesting for various reasons. For one, it is closed under operations of addition $(+)$ and star-product $(*)$ as illustrated in Figure 4 . Adddition is accomplished by placing the tangles side-by-side and attaching the $N E$ strand of the left tangle to the $N W$ strand of the right tangle, while attaching the $S E$ strand of the left tangle to the $S W$ strand of the right tangle. The star product is accomplished by placing one tangle underneath the other and attaching the upper strands of the lower tangle to the lower strands of the upper tangle.

The mirror image of a tangle $T$ is denoted by $-T$ and it is obtained by switching all the crossings in $T$. A third operation illustrated in Figure 4 is inversion, accomplished by turning the tangle counter-clockwise by $90^{\circ}$ in the plane and taking its mirror image. The inverse of a tangle $T$ is denoted by $T^{i}$. It is worth noting that turning the tangle clockwise by $90^{\circ}$ is the cancelling operation, and its result will be denoted by $T^{-i}$. The inversion of a 2 -tangle is an order 4 operation. We also let $T^{r}$ denote a counter-clockwise rotation of $T$ by $90^{\circ}$ in the plane. This is referred to as the rotate of the tangle $T$. Thus inversion is accomplished by rotation and mirror image: $T^{i}=-T^{r}$. The cancelling operation of $T^{r}$ is $T^{-r}$. Remarkably, for rational tangles the inversion is an order 2 operation, i.e. $T^{-i} \sim T^{i}$ and $T \sim\left(T^{i}\right)^{i}$. For this reason we shall also denote the inverse of a 2 -tangle $T$ by $1 / T$, and hence the rotate of the tangle $T$ will be denoted by $-1 / T$. As we shall see later, these notations are harmonious with a method of evaluating a 2 -tangle by a fraction. We note that all operations in $T_{(2,2)}$ can be generalized appropriately to operations in $T_{(m, n)}$. 


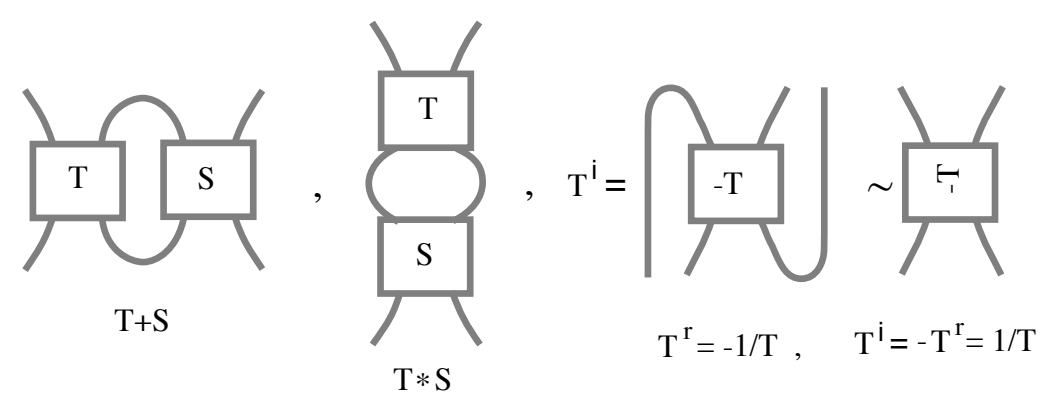

FIGURE 4. Addition, product and inversion of 2-tangles

Finally, the special symmetry of the endpoints of 2-tangles allows for the following closing operations, which yield two different knots: the Numerator of a 2-tangle $T$, denoted by $N(T)$, which is obtained by joining with simple arcs the two upper endpoints and the two lower endpoints of $T$, and the Denominator of a 2 -tangle $T$, which is obtained by joining with simple arcs each pair of the corresponding top and bottom endpoints of $T$, and it shall be denoted by $D(T)$. We have $N(T)=D\left(T^{r}\right)$ and $D(T)=N\left(T^{r}\right)$. We note that every knot or link can be regarded as the numerator closure of a 2 -tangle.

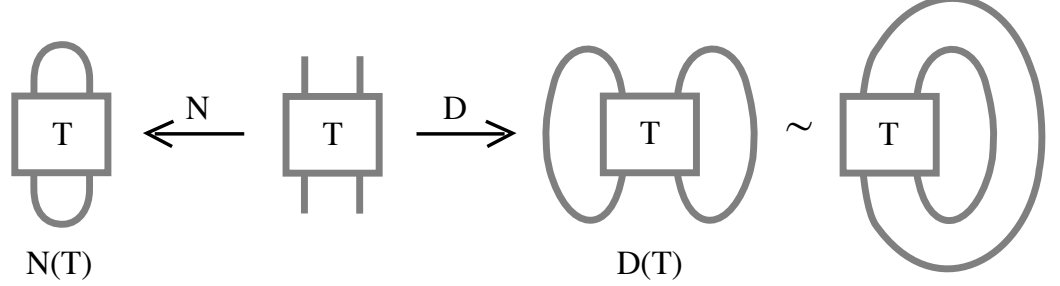

FiguRE 5. The numerator and denominator of a 2-tangle

We obtain $D(T)$ from $N(T)$ by a [0] - [ $\infty$ interchange, as shown in Figure 6. This 'transmutation' of the numerator to the denominator is a precursor to the tangle model of a recombination event in DNA, see Section 9. The $[0]-[\infty]$ interchange can be described algebraically by the equations:

$$
N(T)=N(T+[0]) \longrightarrow N(T+[\infty])=D(T) .
$$

This paper will concentrate on the class of rational knots and links that come from closing the rational tangles. We point out that, even though the sum/product of rational tangles is in general not rational, the numerator (denominator) closure of the sum (product) of two rational tangles is still a rational knot. Another interesting phenomenon is that it may happen that two rational tangles are not isotopic but have isotopic numerators. This is the basic idea behind the classification of rational knots, see Section 5. 


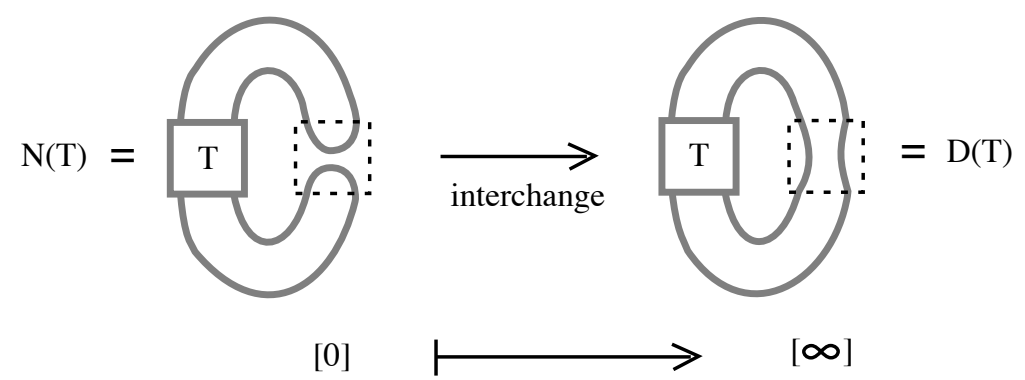

FiguRE 6 . The $[0]-[\infty]$ interchange

Note on the types of crossings. The type of crossings of knots and 2-tangles follow the checkerboard rule: shade the regions of the tangle (knot) in two colors, starting from the left (outside) to the right (inside) with grey, and so that adjacent regions have different colors. Such a shading is illustrated in Figure 7. Crossings in the tangle are said to be of positive type if they are arranged with respect to the shading as exemplified in Figure 7 by the tangle $[+1]$, i.e. they have the region on the right shaded as one walks towards the crossing along the over-arc. Crossings of the reverse type are said to be of negative type and they are exemplified in Figure 7 by the tangle $[-1]$. (Compare with the rational tangle of Figure 1.) The reader should note that our crossing type and sign conventions are the opposite of those in [5]. Our conventions agree with those of Ernst and Sumners [10], which also follow the standard conventions of biologists.

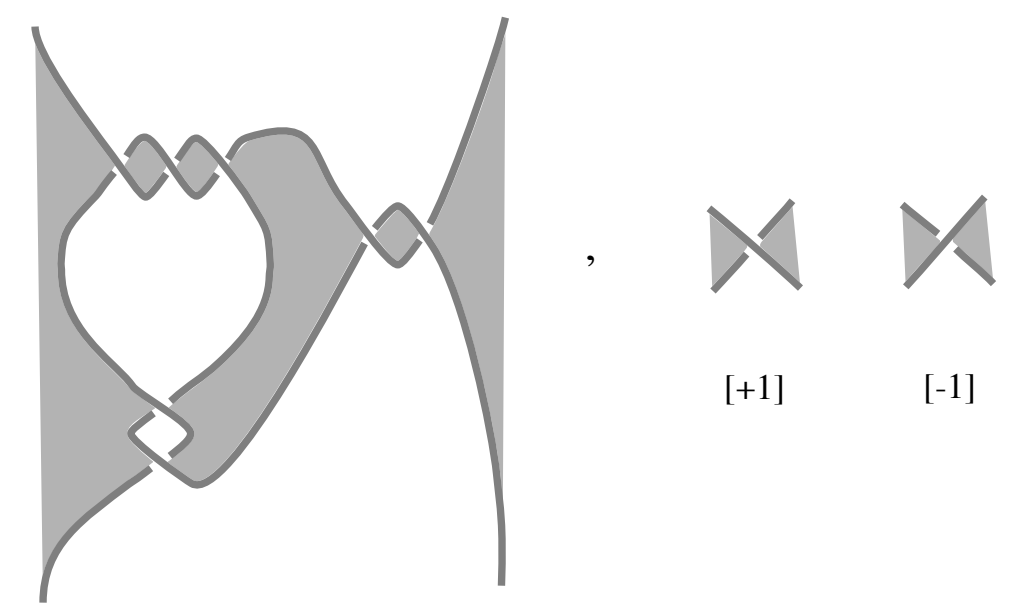

Figure 7 . The checkerboard rule for shading

A tangle is said to be alternating if the crossings in the tangle alternate from under to over as we go along any component or arc of the weave. Similarly, a knot is alternating if it possesses an alternating diagram. Notice that, according to the 
checkerboard shading, the only way the weave alternates is if any two adjacent crossings are of the same type, and this propagates to the whole diagram. Thus, a tangle or a knot diagram is alternating if and only if it has all crossings of the same type.

A flype is an isotopy move applied on a 2 -subtangle of the form $[ \pm 1]+t$ or $[ \pm 1] * t$ and it fixes the endpoints of the subtangle, see Figure 8. A flype preserves the alternating structure of a diagram. Even more, flypes are the only isotopy moves needed in the statement of the celebrated Tait Conjecture for alternating knots, stating that two alternating knots are isotopic if and only if any two corresponding diagrams on $S^{2}$ are related by a finite sequence of flypes. This was posed by P.G. Tait, [37] in 1898 and was proved by W. Menasco and M. Thistlethwaite, [21] in 1993.

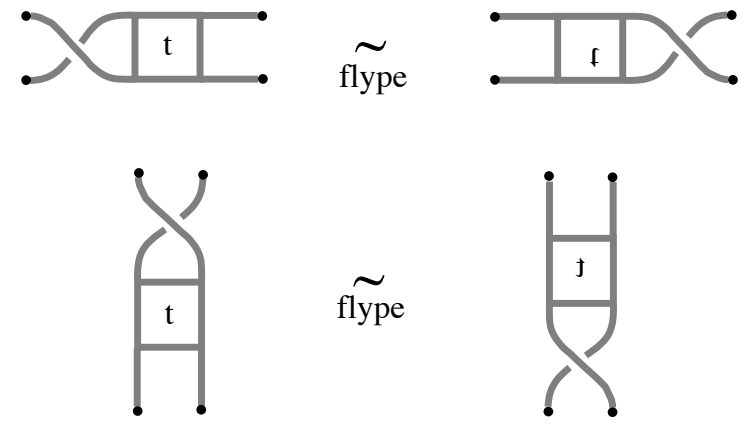

Figure 8 . The flype moves

We describe now another operation applied on rational tangles, which turns out to be an isotopy. We say that $R^{\text {hlip }}$ is the horizontal flip of the tangle $R$ if $R^{\text {flip }}$ is obtained from $R$ by a $180^{\circ}$ rotation around a horizontal axis on the plane of $R$. Moreover, $R^{v f l i p}$ is the vertical flip of the 2-tangle $R$ if $R^{v f l i p}$ is obtained from $R$ by a $180^{\circ}$ rotation around a vertical axis on the plane of $R$. See Figure 9 for illustrations. Note that a flip switches the endpoints of the tangle and, in general, a flipped tangle is not isotopic to the original one. But it is a remarkable property of rational tangles that $T \sim T^{\text {hflip }}$ and $T \sim T^{\text {vflip }}$ for any rational tangle $T$. See [15] for a proof. This is obvious for the tangles $[n]$ and $\frac{1}{[n]}$. Using the vertical flip and induction it is easy to see that the standard and the 3 -strand braid representation of a rational tangle are indeed equivalent.

The above isotopies composed consecutively yield $T \sim\left(T^{i}\right)^{i}=\left(T^{r}\right)^{r}$ for any rational tangle $T$. This says that inversion (rotation) is an operation of order 2 for rational tangles. Thus, the two inverses $T^{i}$ and $T^{-i}$ of a rational tangle $T$ are in fact isotopic, so we can rotate the mirror image of $T$ by $90^{\circ}$ either counterclockwise or clockwise to obtain $T^{i}$. Then, with this notation we have $\frac{1}{\frac{1}{T}}=T$, and this conforms with our notation $\frac{1}{T}$ for the inverse of a 2-tangle, and $T^{r}=\frac{1}{-T}=-\frac{1}{T}$. 

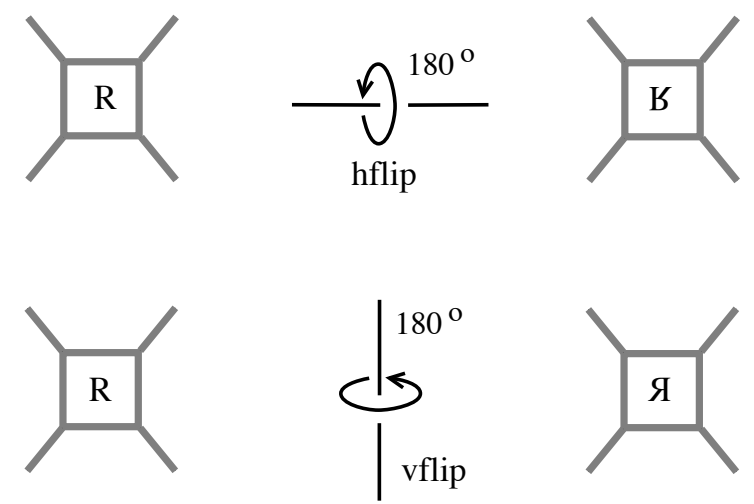

Figure 9. The horizontal and vertical flip

\section{Rational Tangles and their Canonical Form}

In this section we study rational tangles and we show that every rational tangle is isotopic to an alternating one, that is said to be in canonical form. We defined rational tangles as being obtained by applying a finite number of consecutive twists of neighbouring endpoints to the elementary tangles [0] or [ $\infty]$, (recall Definition 2.1). Clearly, the simplest rational tangles are the $[0]$, the $[\infty]$, the $[+1]$ and the $[-1]$ tangles, while the next simplest ones are:

(i) The integer tangles, denoted by $[n]$, made of $n$ horizontal twists, $n \in \mathbb{Z}$.

(ii) The vertical tangles, denoted by $\frac{1}{[n]}$, made of $n$ vertical twists, $n \in \mathbb{Z}$. These are the inverses of the integer tangles, see Figure 10. This terminology explains the need for mirror imaging in the definition of inversion.

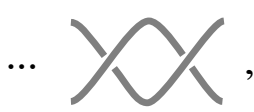

$[-2]$

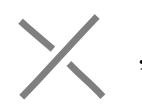

$[-1]$

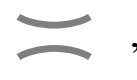

[0]

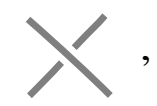

[1]

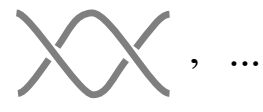

[2]

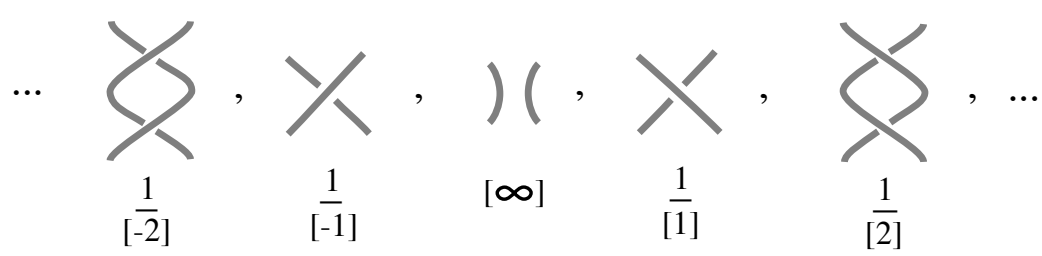

FiguRE 10. The elementary rational tangles

Note that the inverse of a 2-tangle is usually not isotopic to the original tangle, but it is the case that $[+1]^{-1}=[+1]$ and $[-1]^{-1}=[-1]$. Note also that the twists generating the rational tangles could take place between the right, left, top or bottom endpoints of a previously created rational tangle. Using obvious flypes on appropriate subtangles one can always bring the twists to the right or bottom of the 
tangle. We shall then say that the rational tangle is in standard form. For example the rational tangles of Figure 1 and of Figure 7 are in standard form. Hence, a rational tangle in standard form can be obtained inductively from a previously created rational tangle, $T$ say, either by adding an integer tangle on the right: $T \rightarrow T+[ \pm n]$, or by multiplying by a vertical tangle at the bottom: $T \longrightarrow T * \frac{1}{[ \pm n]}$, see Figure 11. For example, Figure 1 illustrates the tangle $\left(\left([3] * \frac{1}{[-2]}\right)+[2]\right)$, while Figure 7 illustrates the tangle $\left(\left([3] * \frac{1}{[2]}\right)+[2]\right)$ in standard form. Equivalently, $a$ rational tangle in standard form is created inductively by consecutive additions of the tangles $[ \pm 1]$ only on the right and multiplications by the tangles $[ \pm 1]$ only at the bottom, starting from the tangles [0] or [ $\infty]$.

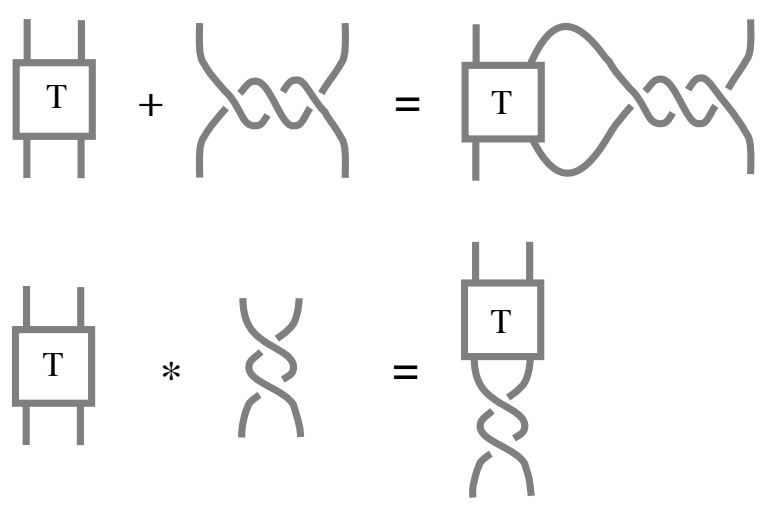

Figure 11. Creating new rational tangles

A rational tangle in standard form has an algebraic expression of the type:

$$
\left(\left(\left(\left[a_{n}\right] * \frac{1}{\left[a_{n-1}\right]}\right)+\left[a_{n-2}\right]\right) * \cdots * \frac{1}{\left[a_{2}\right]}\right)+\left[a_{1}\right], \text { for } a_{2}, \ldots, a_{n} \in \mathbb{Z}-\{0\},
$$

where $\left[a_{1}\right]$ may be $[0]$ and $\left[a_{n}\right]$ may be $[\infty]$ (see also Remark 3.1 below). Figure 12 illustrates two equivalent ways of representing an abstract rational tangle in standard form: the standard representation and the 3-strand-braid representation. This last one is a particular way of closing a three-strand braid. In either representation the rational tangle begins to twist from the tangle $\left[a_{n}\right]\left(\left[a_{5}\right]\right.$ in Figure 12), and it untwists from the tangle $\left[a_{1}\right]$. The 3 -strand-braid representation is actually a compressed version of the vertical flip of the standard representation. Indeed, the upper row of crossings of the 3-strand-braid representation corresponds to the horizontal crossings of the standard representation and the lower row to the vertical ones. Thus, the two representations for rational tangles are equivalent, as it becomes clear from the discussion above about flips. Note that in the 3-strand-braid representation we need to draw the mirror images of the even terms, since when we rotate them to the vertical position we obtain crossings of the opposite type. The 3-strand-braid representation turns out to be more appropriate for studying rational knots. 


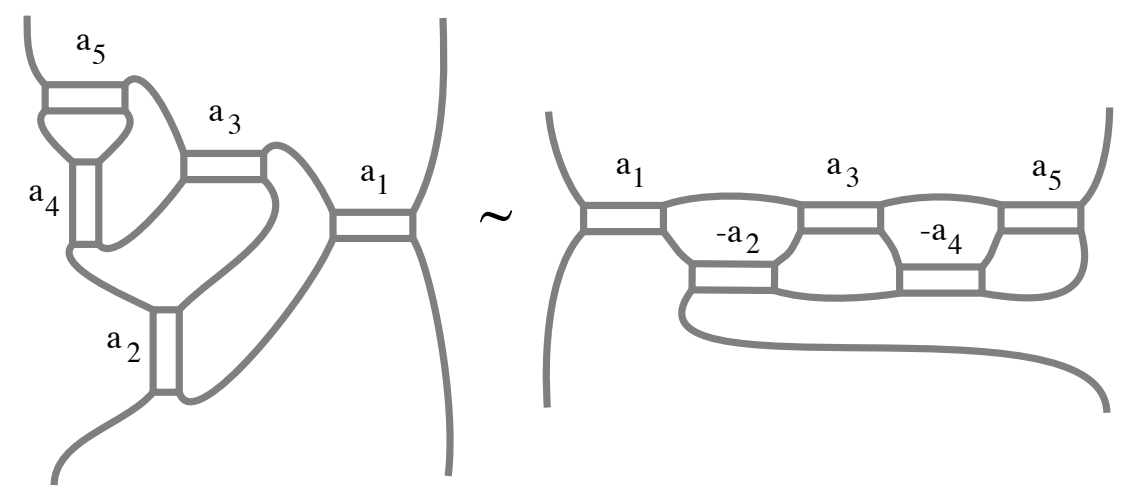

FiguRE 12. The standard and 3-strand-braid representation

Figure 12 illustrates an abstract rational tangle in standard form with an odd number of sets of twists $(n=5)$. Note that if $n$ is even and $\left[a_{1}\right]$ is horizontal then $\left[a_{n}\right]$ has to be vertical. See the left illustration of Figure 13 for such an example of $n$ even.

REMARK 3.1. When we start creating a rational tangle, the very first crossing can be equally seen as a horizontal or as a vertical one. Thus, we may always assume that we start twisting from the [0]-tangle. Moreover, because of the same ambiguity, the number $n$ in the above notation may be assumed to be odd. This is sufficiently illustrated in Figure 13. We shall make this assumption for proving Theorems 1.1, 1.2 and 1.3.

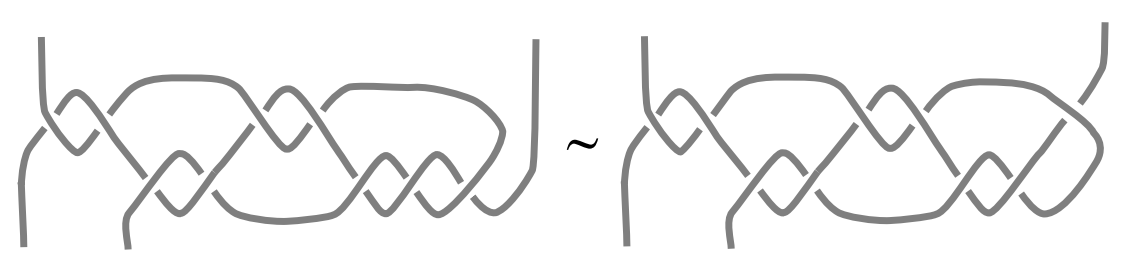

Figure 13. The ambiguity of the first crossing

From the above one may associate to a rational tangle diagram a vector of integers $\left(a_{1}, a_{2}, \ldots, a_{n}\right)$, where the first entry denotes the place where the tangle starts unravelling, and the last entry where it begins to twist. This vector is unique up to breaking the entry $a_{n}$ by a unit, because of Remark 3.1. I.e. $\left(a_{1}, a_{2}, \ldots, a_{n}\right)=$ $\left(a_{1}, a_{2}, \ldots, a_{n}-1,1\right)$, if $a_{n}>0$, and $\left(a_{1}, a_{2}, \ldots, a_{n}\right)=\left(a_{1}, a_{2}, \ldots, a_{n}+1,-1\right)$, if $a_{n}<0$. Thus $n$ may be always assumed to be odd. The example of Figure 1 is associated to the vector $(2,-2,3)$, while the one of Figure 7 is associated to the vector $(2,2,3)$. As we shall soon see, if a rational tangle changes by an isotopy, the associated vector does not necessarily remain the same. 
We next observe that multiplication of a rational tangle $T$ by $\frac{1}{[n]}$ may be obtained as addition of $[n]$ to the inverse $\frac{1}{T}$ followed by inversion. Indeed, we have:

LEMMA 3.2. The following tangle equation holds for any rational tangle $T$.

$$
T * \frac{1}{[n]}=\frac{1}{[n]+\frac{1}{T}} .
$$

Proof. Observe that a $90^{\circ}$ clockwise rotation of $T * \frac{1}{[n]}$ produces $-[n]-\frac{1}{T}$. Hence, from the above $\left(T * \frac{1}{[n]}\right)^{r}=-[n]-\frac{1}{T}$, and thus $\left(T * \frac{1}{[n]}\right)^{i}=[n]+\frac{1}{T}$. So, taking inversions on both sides yields the tangle equation of the statement.

Lemma 3.2 implies that the following two simple algebraic operations between rational tangles preserve the rational tangle structure and, in fact, they generate the whole class of rational tangles: Addition of [1] or [-1] and Inversion. Moreover, it is easy to see that inversion can be replaced by Rotation.

DeFinition 3.3. A continued fraction in integer tangles is an algebraic description of a rational tangle via a continued fraction built from the tangles $\left[a_{1}\right]$, $\left[a_{2}\right], \ldots,\left[a_{n}\right]$ with all numerators equal to 1 , namely an expression of the type:

$$
\left[\left[a_{1}\right],\left[a_{2}\right], \ldots,\left[a_{n}\right]\right]:=\left[a_{1}\right]+\frac{1}{\left[a_{2}\right]+\cdots+\frac{1}{\left[a_{n-1}\right]+\frac{1}{\left[a_{n}\right]}}}
$$

for $a_{2}, \ldots, a_{n} \in \mathbb{Z}-\{0\}$ and $n$ even or odd. We allow that the term $a_{1}$ may be zero, and in this case the tangle [0] may be omitted. A rational tangle described via a continued fraction in integer tangles is said to be in continued fraction form. The length of the continued fraction is arbitrary - in the previous formula illustrated with length $n$-whether the first summand is the tangle [0] or not.

It follows from Lemma 3.2 that inductively every rational tangle can be written in continued fraction form. Lemma 3.2 makes it easy to write out the continued fraction form of a given rational tangle, since horizontal twists are integer additions, and multiplications by vertical twists are the reciprocals of integer additions. For example, Figure 1 illustrates the rational tangle $[2]+\frac{1}{[-2]+\frac{1}{[3]}}$, Figure 7 illustrates the rational tangle $[2]+\frac{1}{[2]+\frac{1}{[3]}}$, while the tangles of Figure 12 both depict the rational tangle $\left[\left[a_{1}\right],\left[a_{2}\right],\left[a_{3}\right],\left[a_{4}\right],\left[a_{5}\right]\right]$. In abstract terms:

$$
\left([c] * \frac{1}{[b]}\right)+[a] \text { has the continued fraction form }[a]+\frac{1}{[b]+\frac{1}{[c]}}=[[a],[b],[c]] .
$$

For $T=\left[\left[a_{1}\right],\left[a_{2}\right], \ldots,\left[a_{n}\right]\right]$ the following statements are now straightforward.

1. $T+[ \pm 1]=\left[\left[a_{1} \pm 1\right],\left[a_{2}\right], \ldots,\left[a_{n}\right]\right]$,

2. $\quad \frac{1}{T}=\left[[0],\left[a_{1}\right],\left[a_{2}\right], \ldots,\left[a_{n}\right]\right]$,

3. $-T=\left[\left[-a_{1}\right],\left[-a_{2}\right], \ldots,\left[-a_{n}\right]\right]$.

Definition 3.4. A rational tangle $T=\left[\left[a_{1}\right],\left[a_{2}\right], \ldots,\left[a_{n}\right]\right]$ is said to be in canonical form if $T$ is alternating and $n$ is odd. 
As an example, the tangle of Figure 7 is in canonical form. Recall that a tangle is alternating if and only if it has crossings all of the same type. Thus, $a$ rational tangle $T=\left[\left[a_{1}\right],\left[a_{2}\right], \ldots,\left[a_{n}\right]\right]$ is alternating if the $a_{i}$ 's are all positive or all negative. For example, the tangle of Figure 7 is alternating. We note that if $T$ is alternating and $n$ even, then we can bring $T$ to canonical form by breaking $a_{n}$ by a unit, i.e. $\left[\left[a_{1}\right],\left[a_{2}\right], \ldots,\left[a_{n}\right]\right]=\left[\left[a_{1}\right],\left[a_{2}\right], \ldots,\left[a_{n}-1\right],[1]\right]$, if $a_{n}>0$, and $\left[\left[a_{1}\right],\left[a_{2}\right], \ldots,\left[a_{n}\right]\right]=\left[\left[a_{1}\right],\left[a_{2}\right], \ldots,\left[a_{n}+1\right],[-1]\right]$, if $a_{n}<0$, recall Remark 3.1. Lemma 3.5 below is a key property of rational tangles.

\section{LEMMA 3.5. Every rational tangle can be isotoped to canonical form.}

Proof. We prove that every rational tangle is isotopic to an alternating tangle. Indeed, if $T$ has a non-alternating continued fraction form then the following configuration, shown in the left of Figure 14, must occur somewhere in $T$, corresponding to a change of sign from one term to an adjacent term in the tangle continued fraction. This configuration is isotopic to a simpler isotopic configuration as shown in that figure.

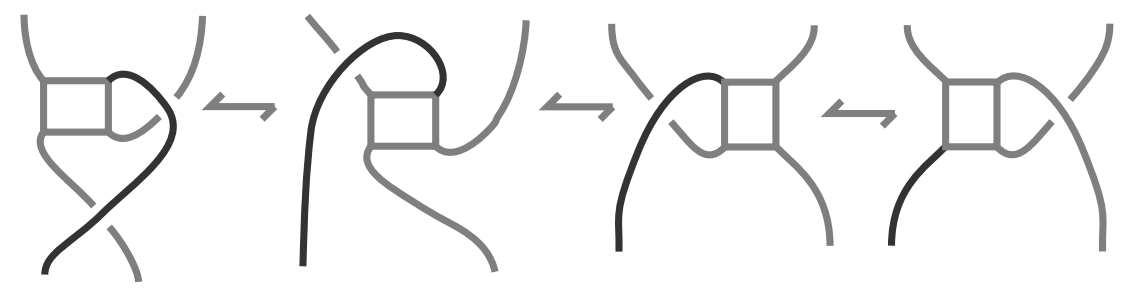

FIGURE 14. Reducing to the alternating form

Therefore, it follows by induction on the number of crossings in the tangle that $T$ is isotopic to an alternating rational tangle. An alternating rational tangle has a continued fraction expression with all terms either positive or negative, and from Remark 3.1 above we may assume that the number of terms is odd.

The alternating nature of the rational tangles will be very useful to us in classifying rational knots and links later in this paper. It turns out from the classification of alternating knots that two alternating tangles are isotopic if and only if they differ by a sequence of flypes. (See [36], [32].) Even more, if the tangles are rational then the 2-subtangle of the flype is also rational, see [15]. It is easy to see that the closure of an alternating rational tangle is an alternating knot. Thus we have

COROLlary 3.6. Rational knots are alternating, since they possess a diagram that is the closure of an alternating rational tangle.

That rational knots are alternating was first proved quite differently by Bankwitz and Schumann and independently by Goeritz, see [1]. 


\section{Continued Fractions and the Classification of Rational Tangles}

In this section we assign to a rational tangle a fraction, and we explore the analogy between rational tangles and continued fractions. This analogy culminates in a common canonical form, which is used to deduce the classification of rational tangles. We need first to recall some facts about continued fractions. The subject of continued fractions is of perennial interest to mathematicians, see for example $[\mathbf{1 8}],[\mathbf{2 4}],[\mathbf{1 9}],[\mathbf{3 8}]$. In this paper we shall only consider continued fractions with all numerators equal to 1 , namely arithmetic expressions of the type

$$
\left[a_{1}, a_{2}, \ldots, a_{n}\right]:=a_{1}+\frac{1}{a_{2}+\cdots+\frac{1}{a_{n-1}+\frac{1}{a_{n}}}}
$$

for $a_{1} \in \mathbb{Z}, a_{2}, \ldots, a_{n} \in \mathbb{Z}-\{0\}$ and $n$ even or odd. As in the case of rational tangles we allow that the term $a_{1}$ may be zero. The length of the continued fraction is the number $n$ whether $a_{1}$ is zero or not. Note that if for $i>1$ all terms are positive or all terms are negative and $a_{1} \neq 0$ or $a_{1}=0$, then the absolute value of the continued fraction is greater (smaller) than one. Clearly, the two simple algebraic operations addition of +1 or -1 and inversion generate inductively the whole class of continued fractions starting from zero. For any rational number $\frac{p}{q}$ the following statements are really straightforward.

1. there are $a_{1} \in \mathbb{Z}, a_{2}, \ldots, a_{n} \in \mathbb{Z}-\{0\}$ such that $\frac{p}{q}=\left[a_{1}, a_{2}, \ldots, a_{n}\right]$,

2. $\underline{p} \pm 1=\left[a_{1} \pm 1, a_{2}, \ldots, a_{n}\right]$

3. $\quad \frac{q}{p}=\left[0, a_{1}, a_{2}, \ldots, a_{n}\right]$,

4. $-\frac{p}{q}=\left[-a_{1},-a_{2}, \ldots,-a_{n}\right]$.

Property 1 above is a consequence of Euclid's algorithm. The algorithm by which Property 1 works is illustrated in the proof of Lemma 4.1 below, see also [18]. The main observation now is the following well-known fact about continued fractions. This is the analogue of Lemma 3.5.

LEMMA 4.1. Every continued fraction $\left[a_{1}, a_{2}, \ldots, a_{n}\right]$ can be transformed to a unique canonical form $\left[\beta_{1}, \beta_{2}, \ldots, \beta_{m}\right]$, where all $\beta_{i}$ 's are positive or all negative integers and $m$ is odd.

Proof. It follows immediately from Euclid's algorithm. We evaluate first $\left[a_{1}, a_{2}, \ldots, a_{n}\right]=$ $\frac{p}{q}$, and using Euclid's algorithm we rewrite $\frac{p}{q}$ in the desired form. We illustrate the proof with an example. Suppose that $\frac{p}{q}=\frac{11}{7}$. Then

$$
\begin{gathered}
\frac{11}{7}=1+\frac{4}{7}=1+\frac{1}{\frac{7}{4}}=1+\frac{1}{1+\frac{3}{4}}=1+\frac{1}{1+\frac{1}{\frac{4}{3}}} \\
=1+\frac{1}{1+\frac{1}{1+\frac{1}{3}}}=[1,1,1,3]=1+\frac{1}{1+\frac{1}{1+\frac{1}{2+\frac{1}{1}}}}=[1,1,1,2,1] .
\end{gathered}
$$

It is the form of odd length that is unique, and any form of even length converts to a form of odd length via the transformations

$$
\begin{aligned}
& {\left[b_{1}, b_{2}, \ldots, b_{k}\right]=\left[b_{1}, b_{2}, \ldots, b_{k}-1,+1\right] \text { for } b_{i} \text { 's positive, or }} \\
& {\left[b_{1}, b_{2}, \ldots, b_{k}\right]=\left[b_{1}, b_{2}, \ldots, b_{k}+1,-1\right] \text { for } b_{i} \text { 's negative. }}
\end{aligned}
$$


This completes the proof.

REMARK 4.2. There is an algorithm that can be applied directly to the initial continued fraction to obtain its canonical form without evaluating it. The point is that this algorithm works in parallel with the algorithm for the canonical form of rational tangles, see [15] for details.

We can now define the fraction of a rational tangle.

Definition 4.3. For a rational tangle $T=\left[\left[a_{1}\right],\left[a_{2}\right], \ldots,\left[a_{n}\right]\right]$ we define the fraction $F(T)$ of $T$ to be the numerical value of the continued fraction obtained by substituting integers for the integer tangles in the expression for $T$, i.e.

$$
F(T):=a_{1}+\frac{1}{a_{2}+\cdots+\frac{1}{a_{n-1}+\frac{1}{a_{n}}}}=\left[a_{1}, a_{2}, \ldots, a_{n}\right],
$$

if $T \neq[\infty]$, and $F([\infty]):=\infty=\frac{1}{0}$, as a formal expression.

Clearly the tangle fraction has the following properties.

1. $F(T+[ \pm 1])=F(T) \pm 1$,

2. $\quad F\left(\frac{1}{T}\right)=\frac{1}{F(T)}$,

3. $F(-T)=-F(T)$.

We are now in position to prove Theorem 1.1, the classification of rational tangles. Proof of Theorem 1.1. We show first that if two rational tangles are isotopic they have the same fraction. We only sketch this part and we refer the reader to our paper [15] for the details. Let $T, S$ be two isotopic rational tangles. We bring $T, S$ to their canonical forms $T^{\prime}, S^{\prime}$ respectively. By Remark 4.2, this corresponds to bringing the initial continued fractions $F(T), F(S)$ to their canonical forms. Now, the tangles $T^{\prime}, S^{\prime}$ are alternating and isotopic, so they differ by a sequence of flypes. Thus, by showing that if two rational tangles differ by a flype they have the same continued fraction, and thus the same fraction, we have completed the one direction of the proof.

Conversely, we show that if two rational tangles have the same fraction they are isotopic. Indeed, let $T=\left[\left[a_{1}\right],\left[a_{2}\right], \ldots,\left[a_{n}\right]\right]$ and $S=\left[\left[b_{1}\right],\left[b_{2}\right], \ldots,\left[b_{m}\right]\right]$ be two rational tangles with $F(T)=F(S)=\frac{p}{q}$. We bring $T, S$ to their canonical forms $T^{\prime}=\left[\left[\alpha_{1}\right],\left[\alpha_{2}\right], \ldots,\left[\alpha_{k}\right]\right]$ and $S^{\prime}=\left[\left[\beta_{1}\right],\left[\beta_{2}\right], \ldots,\left[\beta_{l}\right]\right]$ respectively. From the other direction of the theorem discussed above we have $F\left(T^{\prime}\right)=F(T)=F(S)=$ $F\left(S^{\prime}\right)=\frac{p}{q}$. By Lemma 4.1, the fraction $\frac{p}{q}$ has a unique continued fraction expansion in canonical form, say $\frac{p}{q}=\left[\gamma_{1}, \gamma_{2}, \ldots, \gamma_{r}\right]$. This gives rise to the alternating rational tangle in canonical form $Q=\left[\left[\gamma_{1}\right],\left[\gamma_{2}\right], \ldots,\left[\gamma_{r}\right]\right]$, which is uniquely determined from the vector of integers $\left(\gamma_{1}, \gamma_{2}, \ldots, \gamma_{r}\right)$. We claim that $Q=T^{\prime}$ (and similarly $\left.Q=S^{\prime}\right)$. Indeed, if this were not the case we would have the two different continued fractions in canonical form giving rise to the same rational number: $\left[\alpha_{1}, \alpha_{2}, \ldots, \alpha_{k}\right]=\frac{p}{q}=\left[\gamma_{1}, \gamma_{2}, \ldots, \gamma_{r}\right]$. But this contradicts the uniqueness of the canonical form of continued fractions (Lemma 4.1).

Some comments are now due. Theorem 1.1 says that rational numbers are represented bijectively by rational tangles; their negatives are represented by the mirror images and their inverses by inverses of rational tangles. Adding integers to a 
rational number corresponds to adding integer twists to a rational tangle; but sums of non-integer rational numbers do not correspond to the rational tangles of the sums. Moreover, Theorem 1.1 implies that the canonical form of a rational tangle is unique, since the corresponding canonical form of its continued fraction is unique. Another observation is that in order to bring a rational tangle to its canonival form one simply has to calculate its fraction and express it in canonical form. This canonical form gives rise to an alternating tangle in canonical form which, by Theorem 1.1, is isotopic to the initial one. For example, let $T=[[2],[-3],[5]]$. Then $F(T)=[2,-3,5]=\frac{23}{14}$. But $\frac{23}{14}=[1,1,1,1,4]$, thus $T \sim[[1],[1],[1],[1],[4]]$, and this last tangle is the canonical form of $T$. In [15] we discuss the analogy between rational tangles and continued fractions for infinite continued fractions.

There are, in fact, definitions that associate a rational fraction $F(T)$ (including $0 / 1$ and $1 / 0$ ) to any 2 -tangle $T$ whether or not it is rational. The first definition is due to John Conway in [5] using the Alexander polynomial of the $\operatorname{knots} N(T)$ and $D(T)$. In [13] an alternate definition is given that uses the bracket polynomial of the knots $N(T)$ and $D(T)$, and in [12] the fraction of a tangle is related to the conductance of an associated electrical network. Below we give yet a different definition of the fraction using the coloring method. In all these definitions the fraction is by definition an isotopy invariant of tangles, and we have to show that non-isotopic rational tangles will have different fractions. In the present paper and in $[\mathbf{1 5}]$ the fraction of a rational tangle is defined directly from its combinatorial structure (as originally defined by Conway), and we verify the topological invariance of the fraction using the Tait conjecture.

We conclude this section by giving an alternate definition of the fraction that uses the concept of coloring of knots and tangles. We color the arcs of the knot/tangle with integers, using the basic coloring rule that if two undercrossing arcs colored $\alpha$ and $\gamma$ meet at an overcrossing arc colored $\beta$, then $\alpha+\gamma=2 \beta$. We often think of one of the undercrossing arc colors as determined by the other two colors. Then one writes $\gamma=2 \beta-\alpha$.

It is easy to verify that this coloring method is invariant under the Reidemeister moves in the following sense: Given a choice of coloring for the tangle/knot, there is a way to re-color it each time a Reidemeister move is performed, so that no change occurs to the colors on the external strands of the tangle (so that we still have a valid coloring). This means that a coloring potentially contains topological information about a knot or a tangle. In coloring a knot (and also many non-rational tangles) it is usually necessary to restrict the colors to the set of integers modulo $N$ for some modulus $N$. For example, in Figure 15 it is clear that the color set $\mathbb{Z} / 3 \mathbb{Z}=\{0,1,2\}$ is forced for coloring a trefoil knot. When there exists a coloring of a tangle by integers, so that it is not necessary to reduce the colors over some modulus we shall say that the tangle is integral.

It turns out that every rational tangle is integral: To see this choose two 'colors' for the initial strands (e.g. the colors 0 and 1 ) and color the rational tangle as you create it by successive twisting. We call the colors on the initial strands the starting colors. See Figure 16 for an example. It is important that we start coloring from the initial strands, because then the coloring propagates automatically and uniquely. If one starts from somewhere else, one might get into an edge with an undetermined 

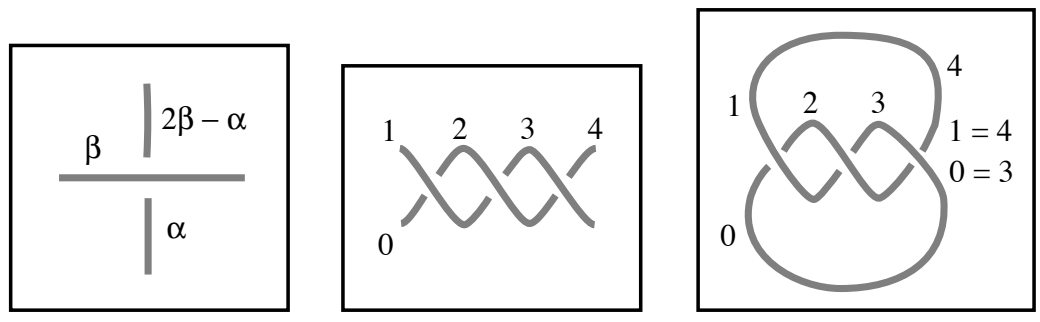

Figure 15. The coloring rule, integral and modular coloring

color. The resulting colored tangle now has colors assigned to its external strands at the northwest, northeast, southwest and southeast positions. Let $N W(T), N E(T)$, $S W(T)$ and $S E(T)$ denote these respective colors of the colored tangle $T$ and define the color matrix of $T, M(T)$, by the equation

$$
M(T)=\left[\begin{array}{cc}
N W(T) & N E(T) \\
S W(T) & S E(T)
\end{array}\right]
$$

Definition 4.4. To a rational tangle $T$ with color matrix $M(T)=\left[\begin{array}{ll}a & b \\ c & d\end{array}\right]$ we associate the number

$$
f(T):=\frac{b-a}{b-d} \in \mathbb{Q} \cup \infty .
$$

It turns out that the entries $a, b, c, d$ of a color matrix of a rational tangle satisfy the 'diagonal sum rule': $a+d=b+c$.
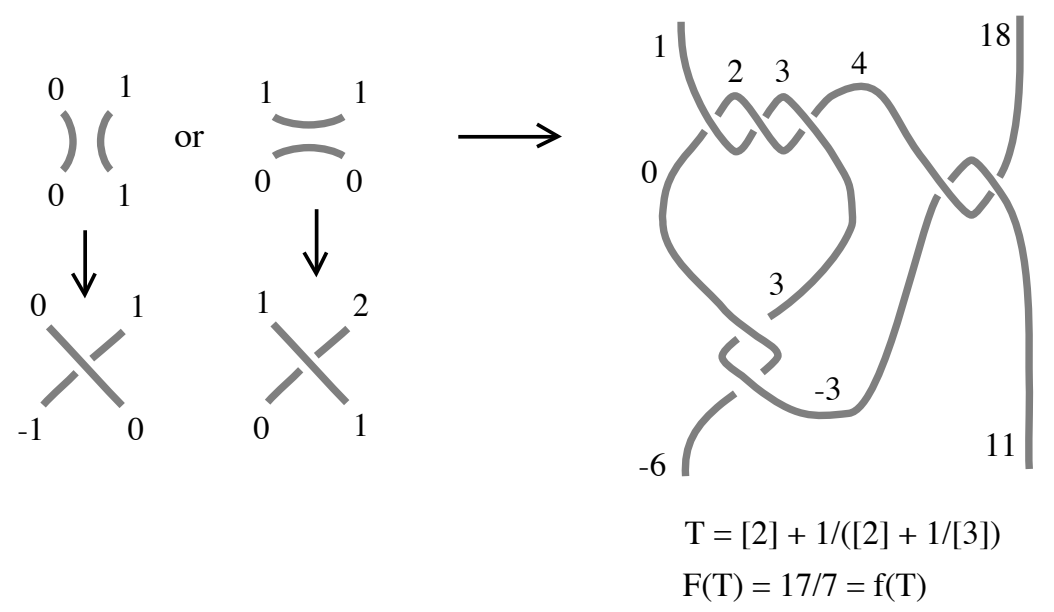

FiguRE 16. Coloring rational tangles

Proposition 4.5. The number $f(T)$ is a topological invariant associated with the tangle $T$. In fact, $f(T)$ has the following properties: 
1. $f(T+[ \pm 1])=f(T) \pm 1$,

2. $f\left(-\frac{1}{T}\right)=-\frac{1}{f(T)}$,

3. $f(-T)=-f(T)$,

4. $\quad f\left(\frac{1}{T}\right)=\frac{1}{f(T)}$,

5. $\quad f(T)=F(T)$.

Thus the coloring fraction is identical to the arithmetical fraction defined earlier.

It is easy to see that $f([0])=\frac{0}{1}, f([\infty])=\frac{1}{0}, f([ \pm 1])= \pm 1$. Hence Statement 5 follows by induction. For proofs of all statements above as well as for a more general set-up we refer the reader to our paper [15]. This definition is quite elementary, but applies only to rational tangles and tangles generated from them by the algebraic operations of ' + ' and ' $*$ '.

In Figure 16 we have illustrated a coloring over the integers for the tangle $[[2],[2],[3]]$ such that every edge is labelled by a different integer. This is always the case for an alternating rational tangle diagram $T$. For the numerator closure $N(T)$ one obtains a coloring in a modular number system. For example in Figure 16 the coloring of $N(T)$ will be in $\mathbb{Z} / 17 \mathbb{Z}$, and it is easy to check that the labels remain distinct in this example. For rational tangles, this is always the case when $N(T)$ has a prime determinant, see $[\mathbf{1 5}]$ and $[\mathbf{2 5}]$.

\section{The Classification of Unoriented Rational Knots}

By taking their numerators or denominators rational tangles give rise to a special class of knots, the rational knots. We have seen so far that rational tangles are directly related to finite continued fractions. We carry this insight further into the classification of rational knots (Schubert's theorems). In this section we consider unoriented knots, and by Remark 3.1 we will be using the 3-strand-braid representation for rational tangles with odd number of terms. Also, by Lemma 3.5 and Corollary 3.6 we may assume all rational knots to be alternating. Note that we only need to take numerator closures, since the denominator closure of a tangle is simply the numerator closure of its rotate.

As already said in the introduction, it may happen that two rational tangles are non-isotopic but have isotopic numerators. The simplest instance of this phenomenon is adding $n$ twists at the bottom of a tangle $T$, see Figure 17. This operation does not change the knot $N(T)$, i.e. $N(T * 1 /[n]) \sim N(T)$, but it does change the tangle, since $F(T * 1 /[n])=F(1 /([n]+1 / T))=1 /(n+1 / F(T))$; so, if $F(T)=p / q$, then $F(T * 1 /[n])=p /(n p+q)$. Hence, if we set $n p+q=q^{\prime}$ we have $q \equiv q^{\prime}(\bmod p)$, just as Theorem 1.2 dictates. Note that reducing all possible bottom twists implies $|p|>|q|$.

Another key example of the arithmetic relationship of the classification of rational knots is illustrated in Figure 18. Here we see that the 'palindromic' tangles

$$
T=[[2],[3],[4]]=[2]+\frac{1}{[3]+\frac{1}{[4]}}
$$




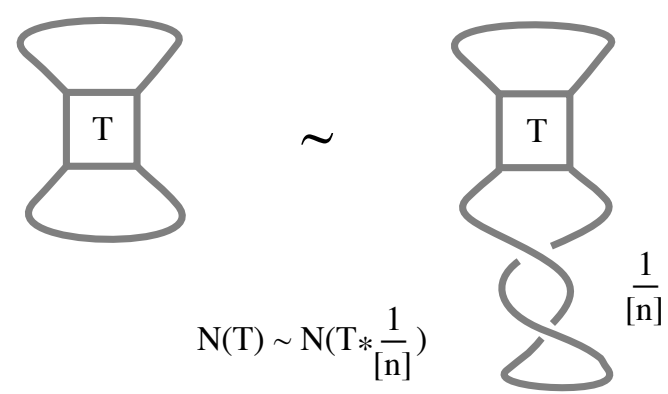

Figure 17. Twisting the bottom of a tangle

and

$$
S=[[4],[3],[2]]=[4]+\frac{1}{[3]+\frac{1}{[2]}}
$$

both close to the same rational knot, shown at the bottom of the figure. The two tangles are different, since they have different corresponding fractions:

$$
F(T)=2+\frac{1}{3+\frac{1}{4}}=\frac{30}{13} \quad \text { and } \quad F(S)=4+\frac{1}{3+\frac{1}{2}}=\frac{30}{7} .
$$

Note that the product of 7 and 13 is congruent to 1 modulo 30 .

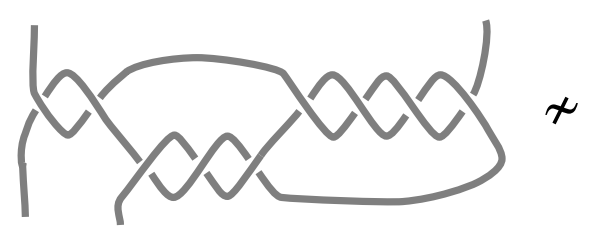

$\mathrm{T}=[2]+1 /([3]+1 /[4])$

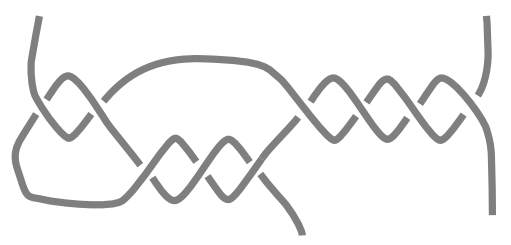

$\mathrm{S}=[4]+1 /([3]+1 /[2])$

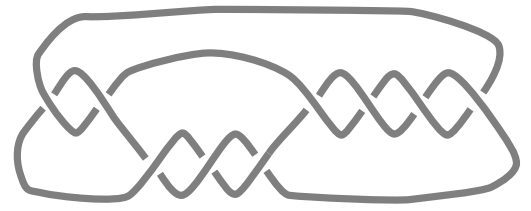

$$
\mathrm{N}(\mathrm{T})=\mathrm{N}(\mathrm{S})
$$

FigURE 18. An instance of the palindrome equivalence

More generally, consider the following two fractions:

$$
F=[a, b, c]=a+\frac{1}{b+\frac{1}{c}} \quad \text { and } \quad G=[c, b, a]=c+\frac{1}{b+\frac{1}{a}} .
$$

We find that 
while

$$
F=a+c \frac{1}{c b+1}=\frac{a b c+a+c}{b c+1}=\frac{P}{Q},
$$

$$
G=c+a \frac{1}{a b+1}=\frac{a b c+c+a}{a b+1}=\frac{P}{Q^{\prime}} .
$$

Thus we found that $F=\frac{P}{Q}$ and $G=\frac{P}{Q^{\prime}}$, where

$$
Q Q^{\prime}=(b c+1)(a b+1)=a b^{2} c+a b+b c+1=b P+1 .
$$

Assuming that $a, b$ and $c$ are integers, we conclude that

$$
Q Q^{\prime} \equiv 1(\bmod P) .
$$

This pattern generalizes to arbitrary continued fractions and their palindromes (obtained by reversing the order of the terms). I.e. If $\left\{a_{1}, a_{2}, \ldots, a_{n}\right\}$ is a collection of $n$ non-zero integers, and if $A=\left[a_{1}, a_{2}, \ldots, a_{n}\right]=\frac{P}{Q}$ and $B=\left[a_{n}, a_{n-1}, \ldots, a_{1}\right]=$ $\frac{P^{\prime}}{Q^{\prime}}$, then $P=P^{\prime}$ and $Q Q^{\prime} \equiv(-1)^{n+1}(\bmod P)$. We will be referring to this as 'the Palindrome Theorem'. The Palindrome Theorem is a known result about continued fractions. For example, see [34] and [15]. Note that we need $n$ to be odd in the previous congruence. This agrees with Remark 3.1 that without loss of generality the terms in the continued fraction of a rational tangle may be assumed to be odd.

Finally, Figure 19 illustrates another basic example for the unoriented Schubert Theorem. The two tangles $R=[1]+\frac{1}{[2]}$ and $S=[-3]$ are non-isotopic by the Conway Theorem, since $F(R)=1+1 / 2=3 / 2$ while $F(S)=-3=3 /-1$. But they have isotopic numerators: $N(R) \sim N(S)$, the left-handed trefoil. Now 2 is congruent to -1 modulo 3 , confirming Theorem 1.2.
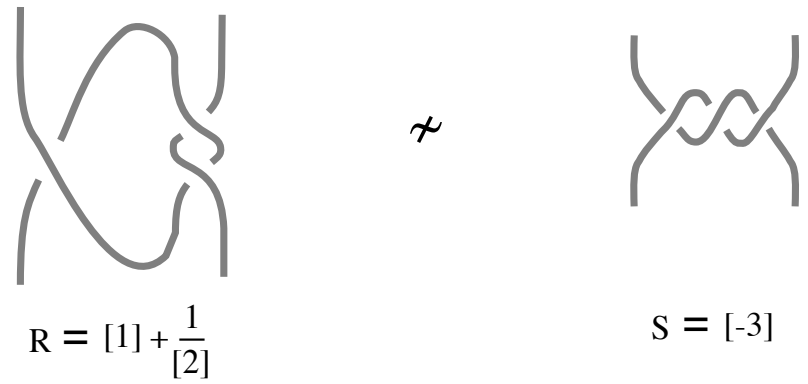

$S=[-3]$

Figure 19. An example of the special cut

We now analyse the above example in general. From the analysis of the bottom twists we can assume without loss of generality that a rational tangle $R$ has fraction $\frac{P}{Q}$, for $|P|>|Q|$. Thus $R$ can be written in the form $R=[1]+T$ or $R=[-1]+T$. We consider the rational knot diagram $K=N([1]+T)$, see Figure 20. (We analyze $N([-1]+T)$ in the same way.) The tangle $[1]+T$ is said to arise as a standard cut on $K$. 


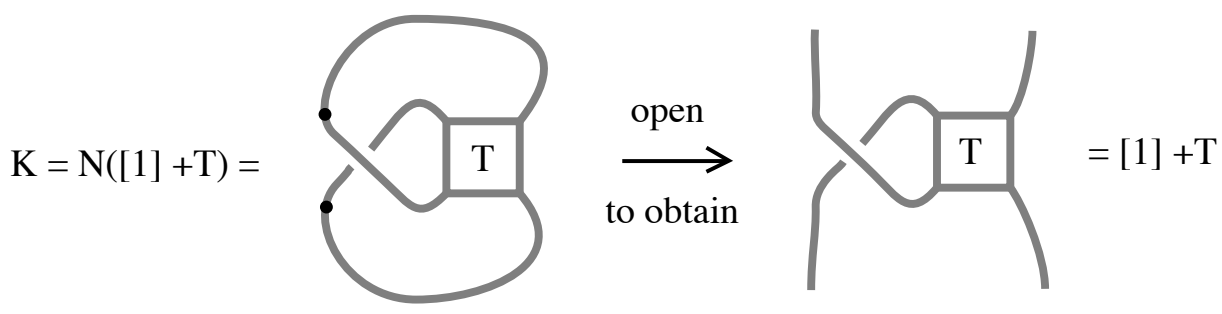

FIgURE 20. A standard cut

Notice that the indicated horizontal crossing of $N([1]+T)$ could be also seen as a vertical one. So, we could also cut the diagram $K$ at the two other marked points (see Figure 21) and still obtain a rational tangle, since $T$ is rational. The tangle obtained by cutting $K$ in this second pair of points is said to arise as a special cut on $K$. Figure 21 demonstrates that the tangle of the special cut is the tangle $[-1]-1 / T$. So we have $N([1]+T) \sim N\left([-1]-\frac{1}{T}\right)$. Suppose now $F(T)=p / q$. Then $F([1]+T)=1+p / q=(p+q) / q$, while $F([-1]-1 / T)=-1-q / p=(p+q) /(-p)$, so the two rational tangles that give rise to the same knot $K$ are not isotopic. Since $-p \equiv q \bmod (p+q)$, this equivalence is another example for Theorem 1.2. In Figure 21 if we took $T=\frac{1}{[2]}$ then $[-1]-1 / T=[-3]$ and we would obtain the example of Figure 19.
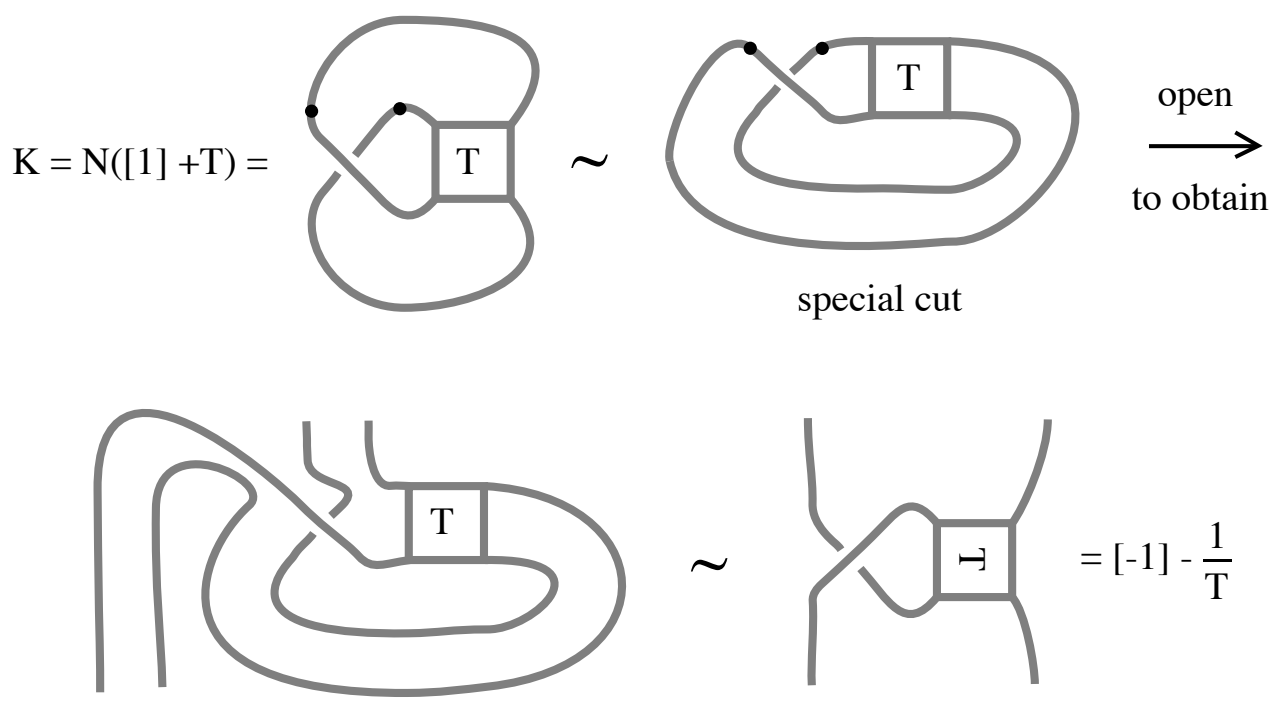

Figure 21. A special cut

The proof of Theorem 1.2 can now proceed in two stages. First, given a rational knot diagram we look for all possible places where we could cut and open it to a rational tangle. The crux of our proof in $[\mathbf{1 6}]$ is the fact that all possible 'rational cuts' on a rational knot fall into one of the basic cases that we have 
already discussed. I.e. we have the standard cuts, the palindrome cuts and the special cuts. In Figure 22 we illustrate on a representative rational knot, all the cuts that exhibit that knot as a closure of a rational tangle. Each pair of points is marked with the same number. The arithmetics is similar to the cases that have been already verified. It is convenient to say that reduced fractions $p / q$ and $p^{\prime} / q^{\prime}$ are arithmetically equivalent, written $p / q \sim p^{\prime} / q^{\prime}$ if $p=p^{\prime}$ and either $q q^{\prime} \equiv 1$ (mod $p)$ or $q \equiv q^{\prime}(\bmod p)$. In this language, Schubert's theorem states that two rational tangles close to form isotopic knots if and only if their fractions are arithmetically equivalent.

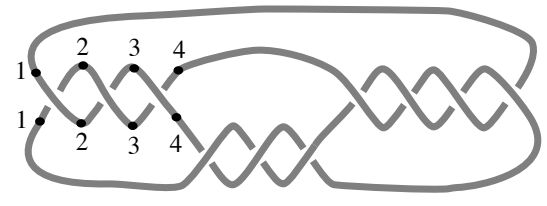

Standard Cuts

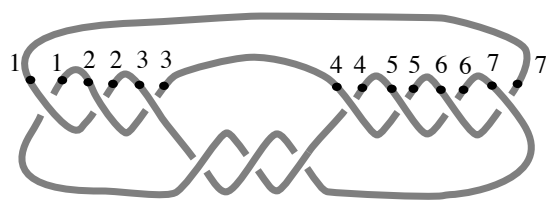

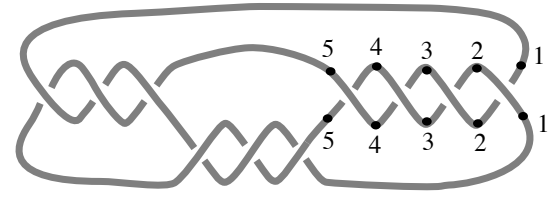

Palindrome Cuts

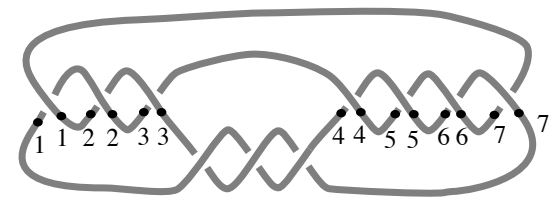

Special Cuts

Figure 22. Standard, palindrome and special cuts

In Figure 23 we illustrate one example of a cut that is not allowed since it opens the knot to a non-rational tangle.
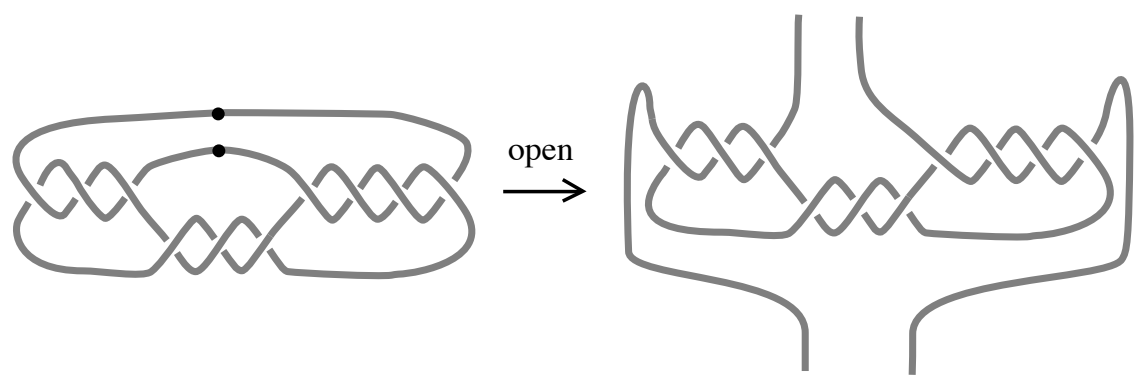

Figure 23. A non-rational cut

In the second stage of the proof we want to check the arithmetic equivalence for two different given knot diagrams, numerators of some rational tangles. By Corollary 3.6 the two knot diagrams may be assumed alternating, so by the Tait Conjecture they will differ by flypes. We analyse all possible flypes to prove that no new cases for study arise. Hence the proof becomes complete at that point. We refer the reader to our paper [16] for the details. 


\section{Rational Knots and Their Mirror Images}

In this section we give an application of Theorem 1.2. An unoriented knot or link $K$ is said to be achiral if it is topologically equivalent to its mirror image $-K$. If a link is not equivalent to its mirror image then it is said be chiral. One then can speak of the chirality of a given knot or link, meaning whether it is chiral or achiral. Chirality plays an important role in the applications of Knot Theory to Chemistry and Molecular Biology. It is interesting to use the classification of rational knots and links to determine their chirality. Indeed, we have the following well-known result (for example see [34] and also page 24, Exercise 2.1.4 in [17]):

THEOREM 6.1. Let $K=N(T)$ be an unoriented rational knot or link, presented as the numerator of a rational tangle $T$. Suppose that $F(T)=p / q$ with $p$ and $q$ relatively prime. Then $K$ is achiral if and only if $q^{2} \equiv-1(\bmod p)$. It follows that achiral rational knots and links are all numerators of rational tangles of the form $\left[\left[a_{1}\right],\left[a_{2}\right], \ldots,\left[a_{k}\right],\left[a_{k}\right], \ldots,\left[a_{2}\right],\left[a_{1}\right]\right]$ for any integers $a_{1}, \ldots, a_{k}$.

Note that in this description we are using a representation of the tangle with an even number of terms. The leftmost twists $\left[a_{1}\right]$ are horizontal, thus the rightmost starting twists $\left[a_{1}\right]$ are vertical.

Proof. With $-T$ the mirror image of the tangle $T$, we have that $-K=N(-T)$ and $F(-T)=p /(-q)$. If $K$ is topologically equivalent to $-K$, then $N(T)$ and $N(-T)$ are equivalent, and it follows from the classification theorem for rational knots that either $q(-q) \equiv 1(\bmod p)$ or $q \equiv-q(\bmod p)$. Without loss of generality we can assume that $0<q<p$. Hence $2 q$ is not divisible by $p$ and therefore it is not the case that $q \equiv-q(\bmod p)$. Hence $q^{2} \equiv-1(\bmod p)$.

Conversely, if $q^{2} \equiv-1(\bmod p)$, then it follows from the Palindrome Theorem that the continued fraction expansion of $p / q$ has to be symmetric with an even number of terms. It is then easy to see that the corresponding rational knot or link, say $K=N(T)$, is equivalent to its mirror image. One rotates $K$ by $180^{\circ}$ in the plane and swings an arc, as Figure 24 illustrates. The point is that the crossings of the second row of the tangle $T$, that are seemingly crossings of opposite type than the crossings of the upper row, become after the turn crossings of the upper row, and so the types of crossings are switched. This completes the proof.

In [9] the authors find an explicit formula for the number of achiral rational knots among all rational knots with $n$ crossings.

\section{The Oriented Case}

Oriented rational knots and links arise as numerator closures of oriented rational tangles. In order to compare oriented rational knots via rational tangles we need to examine how rational tangles can be oriented. We orient rational tangles by choosing an orientation for each strand of the tangle. Here we are only interested in orientations that yield consistently oriented knots upon taking the numerator closure. This means that the two top end arcs have to be oriented one inward and the other outward. Same for the two bottom end arcs. We shall say that two oriented rational tangles are isotopic if they are isotopic as unoriented tangles, by an isotopy that carries the orientation of one tangle to the orientation of the other. Note that, since the end arcs of a tangle are fixed during a tangle isotopy, this means that the 


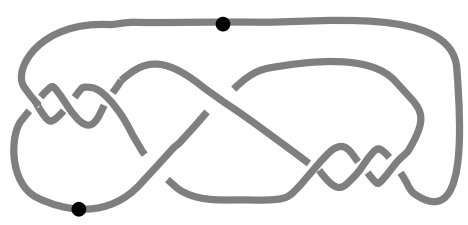

$180^{0}$ rotation
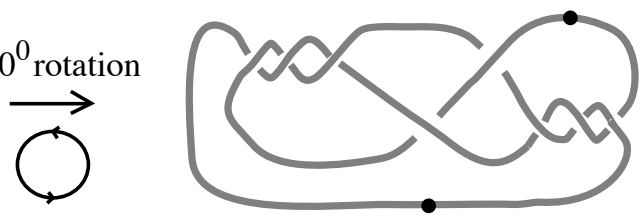

$\mathrm{K}$

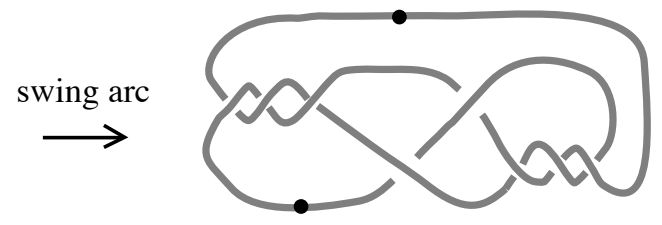

FiguRE 24. An achiral rational link

tangles must have identical orientations at their four end $\operatorname{arcs} N W, N E, S W, S E$. It follows that if we change the orientation of one or both strands of an oriented rational tangle we will always obtain a non-isotopic oriented rational tangle.

Reversing the orientation of one strand of an oriented rational tangle may or may not give rise to isotopic oriented rational knots. Figure 25 illustrates an example of non-isotopic oriented rational knots, which are isotopic as unoriented knots.

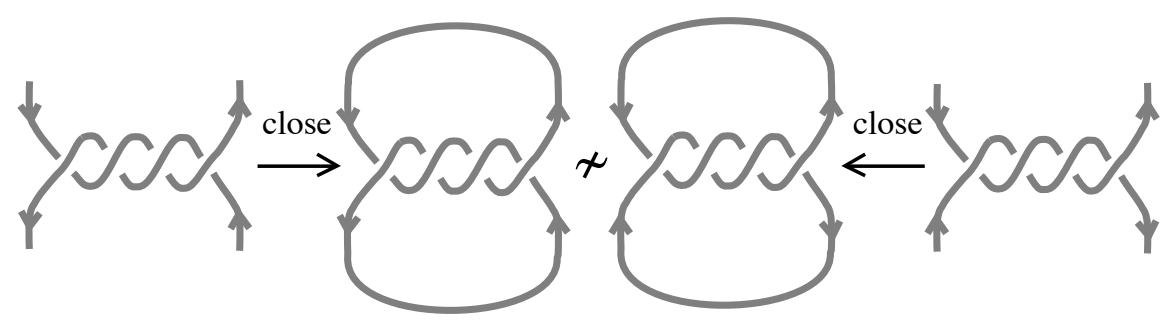

FiguRE 25. Non-isotopic oriented rational links

Reversing the orientation of both strands of an oriented rational tangle will always give rise to two isotopic oriented rational knots or links. We can see this by doing a vertical flip, as Figure 26 demonstrates. Using this observation we conclude that, as far as the study of oriented rational knots is concerned, all oriented rational tangles may be assumed to have the same orientation for their NW and NE end arcs. We fix this orientation to be downward for the $N W$ end arc and upward for the $N E$ arc, as in the examples of Figure 25 and as illustrated in Figure 27. Indeed, if the orientations are opposite of the fixed ones doing a vertical flip the knot may be considered as the numerator of the vertical flip of the original tangle. But this 
is unoriented isotopic to the original tangle (recall Section 3, Figure 9), whilst its orientation pattern agrees with our convention.

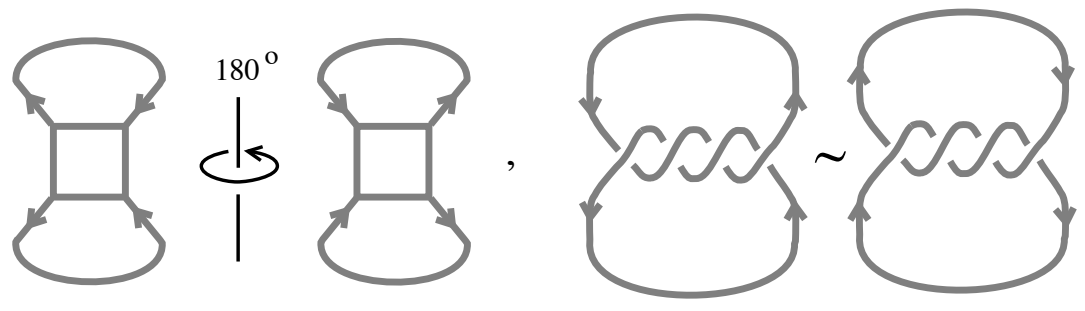

FIGURE 26. Isotopic oriented rational knots and links

Thus we reduce our analysis to two basic types of orientation for the four end arcs of a rational tangle. We shall call an oriented rational tangle of type $I$ if the $S W$ arc is oriented upward and the $S E$ arc is oriented downward, and of type $I I$ if the $S W$ arc is oriented downward and the $S E$ arc is oriented upward, see Figure 27. From the above remarks, any tangle is of type I or type II. Two tangles are said to be compatible it they are both of type I or both of type II and incompatible if they are of different types. In order to classify oriented rational knots seen as numerator closures of oriented rational tangles, we will always compare compatible rational rangles. Note that if two oriented tangles are incompatible, adding a single half twist at the bottom of one of them yields a new pair of compatible tangles, as Figure 27 illustrates. Note also that adding such a twist, although it changes the tangle, it does not change the isotopy type of the numerator closure. Thus, up to bottom twists, we are always able to compare oriented rational tangles of the same orientation type.

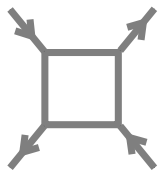

Type I

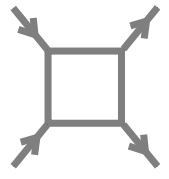

Type II

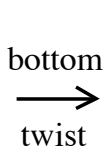

twist
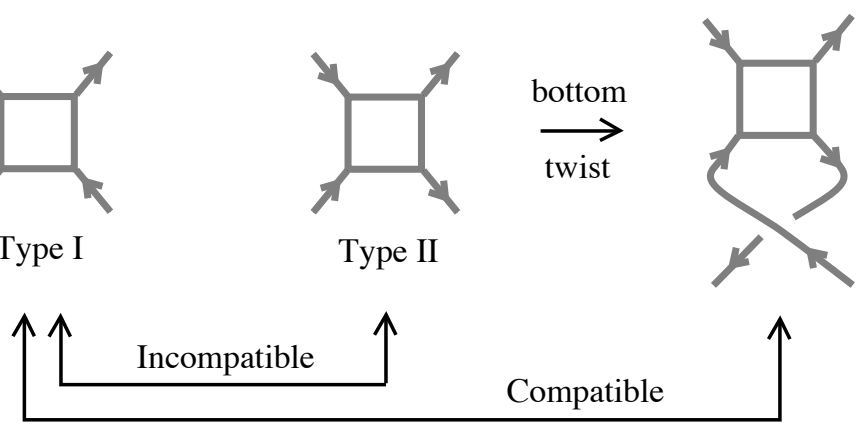

Figure 27. Compatible and incompatible orientations

We shall now introduce the notion of connectivity and we shall relate it to orientation and the fraction of unoriented rational tangles. We shall say that an unoriented rational tangle has connectivity type [0] if the $N W$ end arc is connected 
to the $N E$ end arc and the $S W$ end arc is connected to the $S E$ end arc. Similarly, we say that the tangle has connectivity type $[+1]$ or type $[\infty]$ if the end arc connections are the same as in the tangles $[+1]$ and $[\infty]$ respectively. The basic connectivity patterns of rational tangles are exemplified by the tangles $[0],[\infty]$ and $[+1]$. We can represent them iconically by the symbols shown below.

$$
\begin{gathered}
{[0]=\asymp} \\
{[\infty]=><} \\
{[+1]=\chi}
\end{gathered}
$$

Note that connectivity type [0] yields two-component rational links, while type $[+1]$ or $[\infty]$ yields one-component rational links. Also, adding a bottom twist to a rational tangle of connectivity type [0] will not change the connectivity type of the tangle, while adding a bottom twist to a rational tangle of connectivity type $[\infty]$ will switch the connectivity type to $[+1]$ and vice versa. While the connectivity type of unoriented rational tangles may be $[0],[+1]$ or $[\infty]$, note that an oriented rational tangle of type I will have connectivity type [0] or [ $[$ ] and an oriented rational tangle of type II will have connectivity type $[0]$ or $[+1]$.

Further, we need to keep an accounting of the connectivity of rational tangles in relation to the parity of the numerators and denominators of their fractions. We refer the reader to our paper [16] for a full account.

We adopt the following notation: $e$ stands for even and $o$ stands for odd. The parity of a fraction $p / q$ is defined to be the ratio of the parities ( $e$ or $o$ ) of its numerator and denominator $p$ and $q$. Thus the fraction $2 / 3$ is of parity $e / o$. The tangle [0] has fraction $0=0 / 1$, thus parity $e / o$, the tangle $[\infty]$ has fraction $\infty=1 / 0$, thus parity $o / e$, and the tangle $[+1]$ has fraction $1=1 / 1$, thus parity $o / o$. We then have the following result.

TheOREM 7.1. A rational tangle $T$ has connectivity type $\asymp$ if and only if its fraction has parity e/o. T has connectivity type $><$ if and only if its fraction has parity o/e. $T$ has connectivity type $\chi$ if and only if its fraction has parity o/o. (Note that the formal fraction of $[\infty]$ itself is 1/0.) Thus the link $N(T)$ has two components if and only if $T$ has fraction $F(T)$ of parity $e / o$.

We will now proceed with sketching the proof of Theorem 1.3. We shall prove Schubert's oriented theorem by appealing to our previous work on the unoriented case and then analyzing how orientations and fractions are related. Our strategy is as follows: Consider an oriented rational knot or link diagram $K$ in the form $N(T)$ where $T$ is a rational tangle in continued fraction form. Then any other rational tangle that closes to this knot $N(T)$ is available, up to bottom twists if necessary, as a cut from the given diagram. If two rational tangles close to give $K$ as an unoriented rational knot or link, then there are orientations on these tangles, induced from $K$ so that the oriented tangles close to give $K$ as an oriented knot or link. The two tangles may or may not be compatible. Thus, we must analyze when, comparing with the standard cut for the rational knot or link, another cut produces a compatible or incompatible rational tangle. However, assuming the top orientations are the same, we can replace one of the two incompatible tangles by the tangle obtained by adding a twist at the bottom. It is this possible twist difference that gives rise to the change from modulus $p$ in the unoriented case to 
the modulus $2 p$ in the oriented case. We will now perform this analysis. There are many interesting aspects to this analysis and we refer the reader to our paper [16] for these details. Schubert [33] proved his version of the oriented theorem by using the 2-bridge representation of rational knots and links, see also [4]. We give a tangle-theoretic combinatorial proof based upon the combinatorics of the unoriented case.

The simplest instance of the classification of oriented rational knots is adding an even number of twists at the bottom of an oriented rational tangle $T$, see Figure 27. We then obtain a compatible tangle $T * 1 /[2 n]$, and $N(T * 1 /[2 n]) \sim N(T)$. If now $F(T)=p / q$, then $F(T * 1 /[2 n])=F(1 /([2 n]+1 / T))=1 /(2 n+1 / F(T))=$ $p /(2 n p+q)$. Hence, if we set $2 n p+q=q^{\prime}$ we have $q \equiv q^{\prime}(\bmod 2 p)$, just as the oriented Schubert Theorem predicts. Note that reducing all possible bottom twists implies $|p|>|q|$ for both tangles, if the two tangles that we compare each time are compatible or for only one, if they are incompatible.

We then have to compare the special cut and the palindrome cut with the standard cut. In the oriented case the special cut is the easier to see whilst the palindrome cut requires a more sophisticated analysis. Figure 28 illustrates the general case of the special cut. In order to understand Figure 28 it is necessary to also view Figure 21 for the details of this cut.

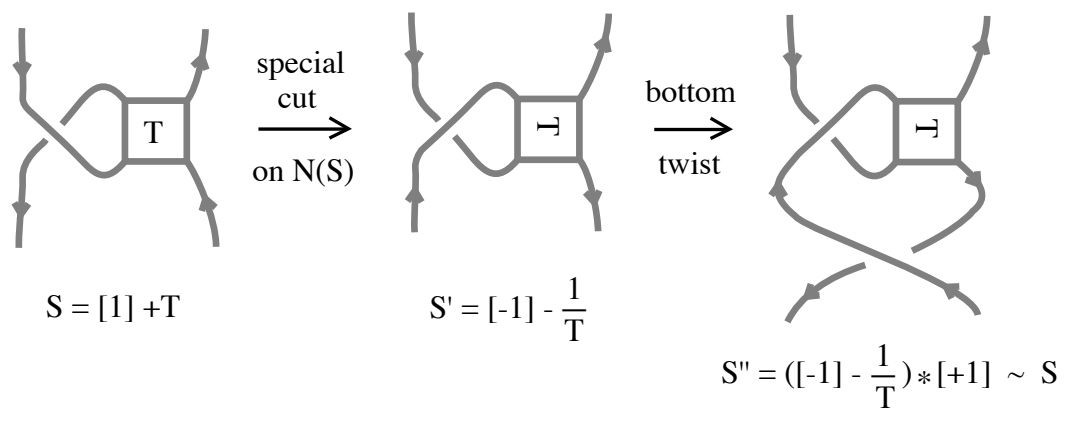

Figure 28. The oriented special cut

Recall that if $S=[1]+T$ then the tangle of the special cut on the knot $N([1]+T)$ is the tangle $S^{\prime}=[-1]-\frac{1}{T}$. And if $F(T)=p / q$ then $F([1]+T)=\frac{p+q}{q}$ and $F\left([-1]-\frac{1}{T}\right)=\frac{p+q}{-p}$. Now, the point is that the orientations of the tangles $S$ and $S^{\prime}$ are incompatible. Applying a $[+1]$ bottom twist to $S^{\prime}$ yields $S^{\prime \prime}=\left([-1]-\frac{1}{T}\right) *[1]$, and we find that $F\left(S^{\prime \prime}\right)=\frac{p+q}{q}$. Thus, the oriented rational tangles $S$ and $S^{\prime \prime}$ have the same fraction and by Theorem 1.1 and their compatibility they are oriented isotopic and the arithmetics of Theorem 1.3 is straightforward.

We are left to examine the case of the palindrome cut. In Figure 29 we illustrate the standard and palindrome cuts on the oriented rational knot $K=N(T)=N\left(T^{\prime}\right)$ where $T=[[2],[1],[2]]$ and $T^{\prime}$ its palindrome. As we can see, the two cuts place incompatible orientations on the tangles $T$ and $T^{\prime}$. Adding a twist at the bottom of $T^{\prime}$ produces a tangle $T^{\prime \prime}=T^{\prime} *[-1]$ that is compatible with $T$. Now we compute 
$F(T)=F\left(T^{\prime}\right)=8 / 3$ and $F\left(T^{\prime \prime}\right)=F\left(T^{\prime} *[-1]\right)=8 /-5$ and we notice that $3 \cdot(-5) \equiv 1(\bmod 16)$ as Theorem 1.3 predicts. This example also illustrates an example of strong invertibility, as we shall see in the next section.
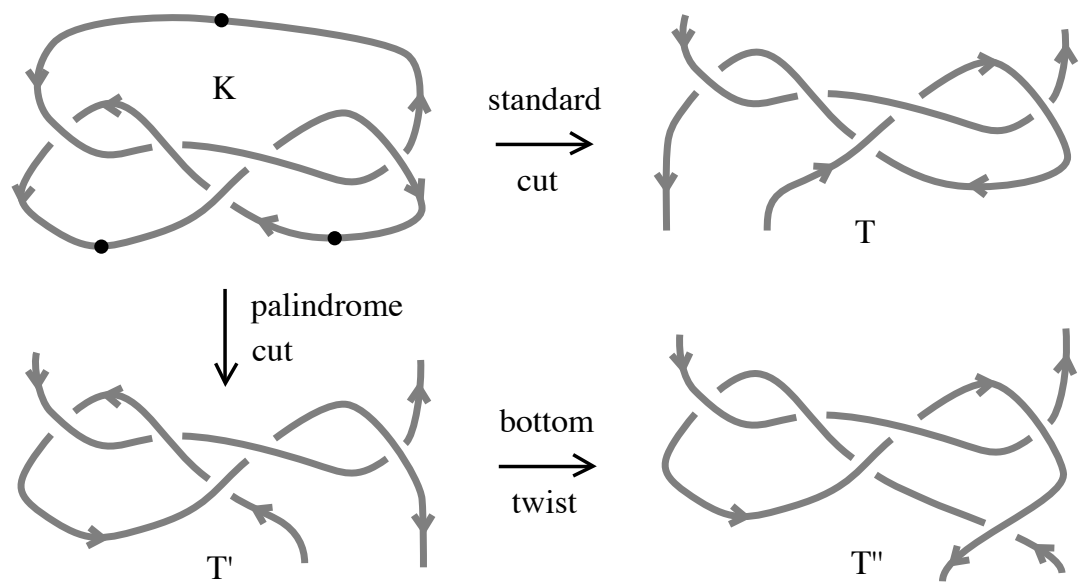

FiguRE 29. Oriented standard cut and palindrome cut

In order to analyze the palindrome case we must understand when the standard cut and the palindrome cut are compatible or incompatible. Then we must compare their respective fractions. This involves a deeper analysis along the lines of Theorem 7.1. More precisely, let $K=N(T)$ be an oriented rational knot or link with $T$ a rational tangle in 3 -strand-braid form. Then the three strands connect according to one of the six permutations of three points, as the first column of Figure 30 illustrates. This is the connectivity chart of the tangle $T$ or the link $K$. For each case we specify by an ' $i$ ' or ' $c$ ' if the standard and the palindrome cut are orientation incompatible or compatible. In the second and third column of the same figure we give the connectivity type and the parity of the standard cut and the palindrome cut of the connectivity chart respectively. We analyze the relation between connectivity, parity and compatibility in the standard and palindrome cuts on $K$. The proof of Theorem 1.3 follows after this analysis by a combination of enumeration and mathematical induction. In particular, we can assume that $K=N\left(\left[\left[a_{1}\right], \ldots,\left[a_{n}\right]\right]\right)$ with $n$ odd. We then know that the matrix product

$$
M=M\left(a_{1}\right) M\left(a_{2}\right) \cdots M\left(a_{n}\right)=\left(\begin{array}{cc}
p & q^{\prime} \\
q & u
\end{array}\right)
$$

encodes the fractions of the tangle $T=\left[\left[a_{1}\right], \ldots,\left[a_{n}\right]\right]$ and its palindrome $T^{\prime}=$ $\left[\left[a_{n}\right], \ldots,\left[a_{1}\right]\right]$ with $F(T)=p / q$ and $F\left(T^{\prime}\right)=p / q^{\prime}$. By construction, $T$ is the standard cut on $K$ and $T^{\prime}$ is the palindrome cut on $K$. Since $\operatorname{Det}(M)=-1$, we have the formula $q q^{\prime}=1+u p$ relating the denominators of these fractions. When $p$ is odd the argument follows from the information on the connectivity chart, Figure 30. When $p$ is even we make an induction argument using the connectivity chart. We use induction to show in this case that 


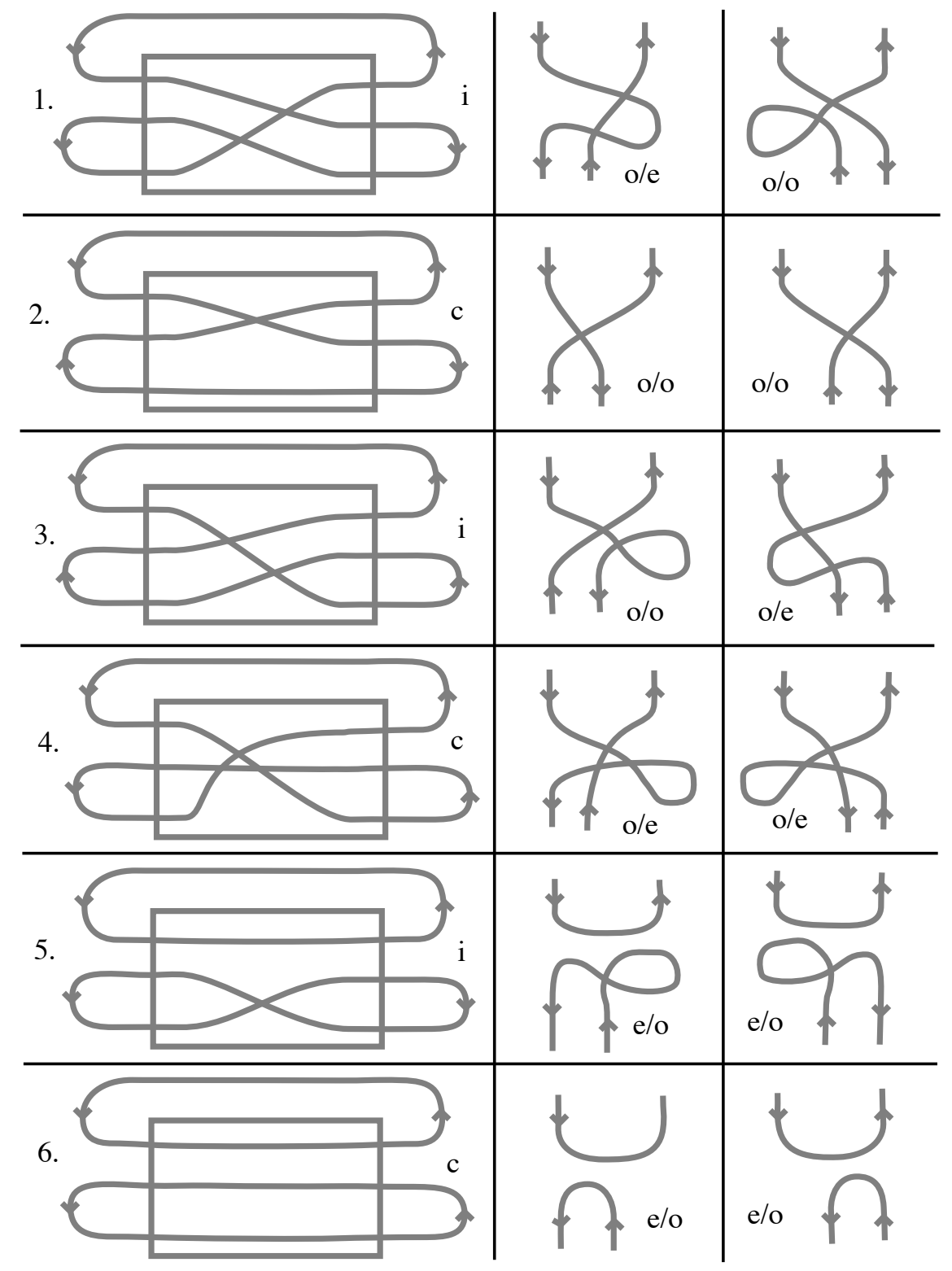

FIgURE 30. The connectivity charts, compatibility and parity

1. $u$ is even if and only if the standard and palindrome cuts are compatible.

2. $u$ is odd if and only if the standard and palindrome cuts are incompatible.

We refer the reader to our paper [16] for the details. The proof sketch of Theorem 1.3 is now complete. 


\section{Strongly Invertible Links}

An oriented knot or link is said to be invertible if it is oriented isotopic to the link obtained from it by reversing all orientations of all components. By applying a vertical rotation by $180^{\circ}$ we have seen that rational knots and links are invertible. A link $L$ of two components is said to be strongly invertible if $L$ is ambient isotopic to itself with the orientation of only one component reversed. This terminology for links is not to be confused with the corresponding terminology for knots in [2]. In Figure 29 we illustrate the $\operatorname{link} L=N([[2],[1],[2]])$. This is a strongly invertible link as is apparent by a $180^{\circ}$ vertical rotation. This link is well-known as the Whitehead link, a link with linking number zero. Note that since [[2], [1], [2]] has fraction equal to $1+1 /(1+1 / 2)=8 / 3$ this link is non-trivial via the classification of rational knots and links. Note also that $3 \cdot 3=1+1 \cdot 8$. In general we have the following.

THEOREM 8.1. Let $L=N(T)$ be an oriented rational link with associated tangle fraction $F(T)=p / q$ of parity $e / o$, with $p$ and $q$ relatively prime and $|p|>|q|$. Then $L$ is strongly invertible if and only if $q^{2}=1+$ up with $u$ an odd integer. It follows that strongly invertible links are all numerators of rational tangles of the form $\left[\left[a_{1}\right],\left[a_{2}\right], \ldots,\left[a_{k}\right],[\alpha],\left[a_{k}\right], \ldots,\left[a_{2}\right],\left[a_{1}\right]\right]$ for any integers $a_{1}, \ldots, a_{k}, \alpha$.

Proof. In $T$ the upper two strands close to form one component of $L$ and the lower two strands close to form the other component of $L$. Let $T^{\prime}$ denote the tangle obtained from the oriented tangle $T$ by reversing the orientation of the component containing the lower two arcs and let $N\left(T^{\prime}\right)=L^{\prime}$. Note that $T$ and $T^{\prime}$ are incompatible. Thus, in order to apply the Schubert Theorem for comparing the links $L$ and $L^{\prime}$ we need to add a bottom twist on $T^{\prime}$. Since $T$ and $T^{\prime}$ have the same fraction $p / q$, after adding the twist we need to compare the fractions $p / q$ and $p /(p+q)$. Since $q$ is not congruent to $(p+q)$ modulo $2 p$, we need to determine when $q(p+q)$ is congruent to 1 modulo $2 p$. This will happen exactly when $q p+q^{2}=1+2 m p$ for some integer $m$. The last equation is the same as saying that $q^{2}=1+u p$ with $u$ odd, since $q$ is odd. It then follows from the Palindrome Theorem for continued fractions that the continued fraction expansion of $p / q$ has to be symmetric with an odd number of terms. It is then easy to see that the corresponding rational link is ambient isotopic to itself through a vertical $180^{\circ}$ rotation, just as in the example of the Whitehead link given above. Hence it is strongly invertible. This completes the proof.

Figure 31 illustrates another example of a strongly invertible rational link. Here $L=N([[3],[1],[1],[1],[3]])=N(T)$. We find $F(T)=40 / 11$ and we observe that $11^{2}=1+3 \cdot 40$.

\section{Applications to the Topology of DNA}

DNA supercoils, replicates and recombines with the help of certain enzymes. Site-specific recombination is one of the ways nature alters the genetic code of an organism, either by moving a block of DNA to another position on the molecule or by integrating a block of alien DNA into a host genome. For a closed molecule of DNA a global picture of the recombination would be as shown in Figure 32, where double-stranded DNA is represented by a single line and the recombination sites are marked with points. This picture can be interpreted as $N(S+[0]) \longrightarrow N(S+[1])$, 


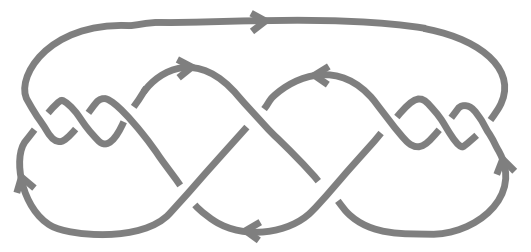

$\mathrm{L}=\mathrm{N}([[3],[1],[1],[1],[3]])$

Figure 31 . An example of a strongly invertible link

for $S=\frac{1}{[-3]}$ in this example. This operation can be repeated as in Figure 33. Note that the $[0]-[\infty]$ interchange of Figure 6 can be seen as the first step of the process.

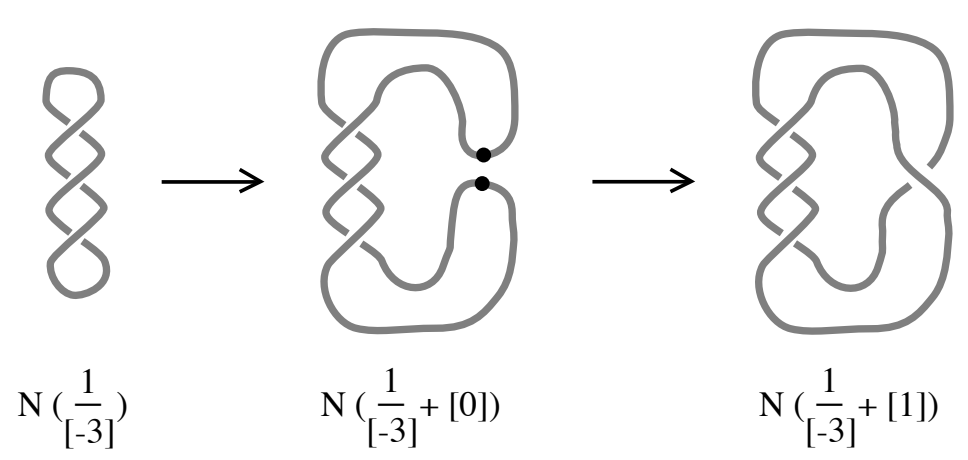

FiguRE 32. Global picture of recombination

In this depiction of recombination, we have shown a local replacement of the tangle [0] by the tangle [1] connoting a new cross-connection of the DNA strands. In general, it is not known without corroborating evidence just what the topological geometry of the recombination replacement will be. Even in the case of a single half-twist replacement such as [1], it is certainly not obvious beforehand that the replacement will always be $[+1]$ and not sometimes the reverse twist of $[-1]$. It was at the juncture raised by this question that a combination of topological methods in biology and a tangle model using knot theory developed by C.Ernst and D.W. Sumners resolved the issue in some specific cases. See [10], [35] and references therein.

On the biological side, methods of protein coating developed by N. Cozzarelli, S.J. Spengler and A. Stasiak et al. In [6] it was made possible for the first time to see knotted DNA in an electron micrograph with sufficient resolution to actually identify the topological type of these knots. The protein coating technique made it possible to design an experiment involving successive DNA recombinations and to examine the topology of the products. In [6] the knotted DNA produced by such successive recombinations was consistent with the hypothesis that all recombinations were of the type of a positive half twist as in $[+1]$. Then D.W. Sumners 


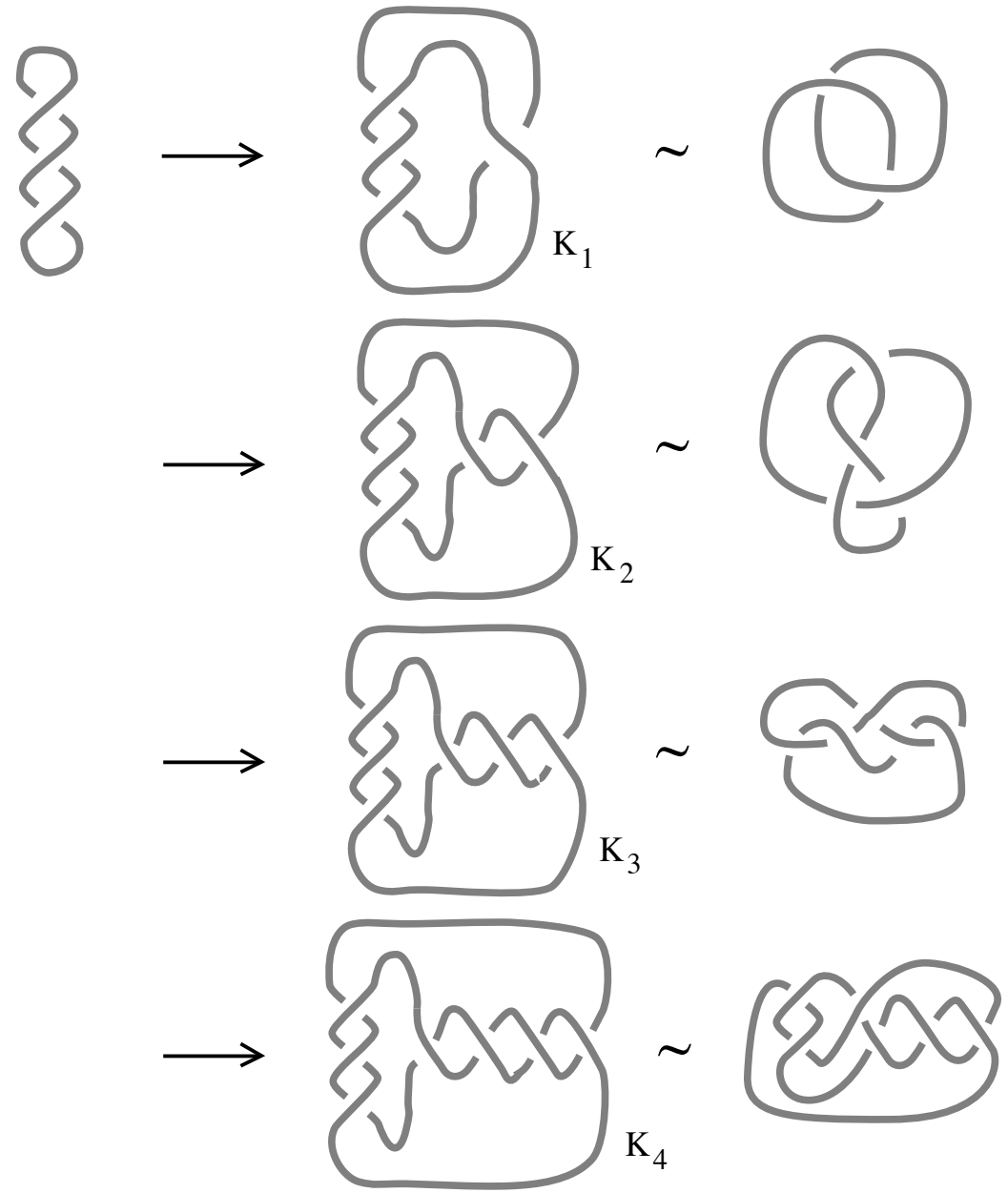

FiguRE 33. Multiple recombinations

and C. Ernst $[\mathbf{1 0}]$ proposed a tangle model for successive DNA recombinations and showed, in the case of the experiments in question, that there was no other topological possibility for the recombination mechanism than the positive half twist $[+1]$. This constituted a unique use of topological mathematics as a theoretical underpinning for a problem in molecular biology.

Here is a brief description of the tangle model for DNA recombination. It is assumed that the initial state of the DNA is described as the numerator closure $N(S)$ of a substrate tangle $S$. The local geometry of the recombination is assumed to be described by the replacement of the tangle [0] with a specific tangle $R$. The results of the successive rounds of recombination are the knots and links

$$
N(S+R)=K_{1}, \quad N(S+R+R)=K_{2}, \quad N(S+R+R+R)=K_{3}, \quad \ldots
$$


Knowing the knots $K_{1}, K_{2}, K_{3}, \ldots$ one would like to solve the above system of equations with the tangles $S$ and $R$ as unknowns. For such experiments Ernst and Sumners [10] used the classification of rational knots in the unoriented case, as well as results of Culler, Gordon, Luecke and Shalen [7] on Dehn surgery to prove that the solutions $S+n R$ must be rational tangles. One could then apply the theorem on the classification of rational knots to deduce (in these instances) the uniqueness of $S$ and $R$. Note that, in these experiments, the substrate tangle $S$ was also pinpointed by the sequence of knots and links that resulted from the recombination.

Here we shall solve tangle equations like the above under rationality assumptions on all tangles in question. This allows us to use only the mathematical techniques developed in this paper. We shall illustrate how a sequence of rational knots and links

$$
N(S+n R)=K_{n}, \quad n=0,1,2,3, \ldots
$$

with $S$ and $R$ rational tangles, such that $R=[r], F(S)=\frac{p}{q}$ and $p, q, r \in \mathbb{Z}(p>0)$ determines $\frac{p}{q}$ and $r$ uniquely if we know sufficiently many $K_{n}$. We call this the "DNA Knitting Machine Analysis".

THEOREM 9.1. Let a sequence $K_{n}$ of rational knots and links be defined by the equations $K_{n}=N(S+n R)$ with specific integers $p, q, r(p>0)$, where $R=$ $[r], F(S)=\frac{p}{q}$. Then $\frac{p}{q}$ and $r$ are uniquely determined if one knows the topological type of the unoriented links $K_{0}, K_{1}, \ldots, K_{N}$ for any integer $N \geq|q|-\frac{p}{q r}$.

Proof. In this proof we shall write $N\left(\frac{p}{q}+n r\right)$ or $N\left(\frac{p+q n r}{q}\right)$ for $N(S+n R)$. We shall also write $K=K^{\prime}$ to mean that $K$ and $K^{\prime}$ are isotopic links. Moreover we shall say for a pair of reduced fractions $P / q$ and $P / q^{\prime}$ that $q$ and $q^{\prime}$ are arithmetically related relative to $P$ if either $q \equiv q^{\prime}(\bmod P)$ or $q q^{\prime} \equiv 1(\bmod P)$. Suppose the integers $p, q, r$ give rise to the sequence of links $K_{0}, K_{1}, \ldots$. Suppose there is some other triple of integers $p^{\prime}, q^{\prime}, r^{\prime}$ that give rise to the same sequence of links. We will show uniqueness of $p, q, r$ under the conditions of the theorem. We shall say "the equality holds for $n$ " to mean that $N((p+q r n) / q)=N\left(\left(p^{\prime}+q^{\prime} r^{\prime} n\right) / q^{\prime}\right)$. We suppose that $K_{n}=N((p+q r n) / q)$ as in the hypothesis of the theorem, and suppose that there are $p^{\prime}, q^{\prime}, r^{\prime}$ such that for some $n$ (or a range of values of $n$ to be specified below) $K_{n}=N\left(\left(p^{\prime}+q^{\prime} r^{\prime} n\right) / q^{\prime}\right)$.

If $n=0$ then we have $N(p / q)=N\left(p^{\prime} / q^{\prime}\right)$. Hence by the classification theorem we know that $p=p^{\prime}$ and that $q$ and $q^{\prime}$ are arithmetically related. Note that the same argument shows that if the equality holds for any two consecutive values of $n$, then $p=p^{\prime}$. Hence we shall assume henceforth that $p=p^{\prime}$. With this assumption in place, we see that if the equality holds for any $n \neq 0$ then $q r=q^{\prime} r^{\prime}$. Hence we shall assume this as well from now on.

If $|p+q r n|$ is sufficiently large, then the congruences for the arithmetical relation of $q$ and $q^{\prime}$ must be equalities over the integers. Since $q q^{\prime}=1$ over the integers can hold only if $q=q^{\prime}=1$ or -1 we see that it must be the case that $q=q^{\prime}$ if the equality is to hold for sufficiently large $n$. From this and the equation $q r=q^{\prime} r^{\prime}$ it follows that $r=r^{\prime}$. It remains to determine a bound on $n$. In order to be sure that $|p+q r n|$ is sufficiently large, we need that $\left|q q^{\prime}\right| \leq|p+q r n|$. Since $q^{\prime} r^{\prime}=q r$, we also know that $\left|q^{\prime}\right| \leq|q r|$. Hence $n$ is sufficiently large if $\left|q^{2} r\right| \leq|p+q r n|$. 
If $q r>0$ then, since $p>0$, we are asking that $\left|q^{2} r\right| \leq p+q r n$. Hence

$$
n \geq\left(\left|q^{2} r\right|-p\right) /(q r)=|q|-(p / q r) .
$$

If $q r<0$ then for $n$ large we will have $|p+q r n|=-p-q r n$. Thus we want to solve $\left|q^{2} r\right| \leq-p-q r n$, whence

$$
n \geq\left(\left|q^{2} r\right|+p\right) /(-q r)=|q|-(p / q r) .
$$

Since these two cases exhaust the range of possibilities, this completes the proof of the theorem.

Here is a special case of Theorem 9.1. See Figure 33. Suppose that we were given a sequence of knots and links $K_{n}$ such that

$$
K_{n}=N\left(\frac{1}{[-3]}+[1]+[1]+\ldots+[1]\right)=N\left(\frac{1}{[-3]}+n[1]\right) .
$$

We have $F\left(\frac{1}{[-3]}+n[1]\right)=(3 n-1) / 3$ and we shall write $K_{n}=N([(3 n-1) / 3])$. We are told that each of these rational knots is in fact the numerator closure of a rational tangle denoted

$$
[p / q]+n[r]
$$

for some rational number $p / q$ and some integer $r$. That is, we are told that they come from a DNA knitting machine that is using rational tangle patterns. But we only know the knots and the fact that they are indeed the closures for $p / q=-1 / 3$ and $r=1$. By this analysis, the uniqueness is implied by the knots and links $\left\{K_{1}, K_{2}, K_{3}, K_{4}\right\}$. This means that a DNA knitting machine $K_{n}=N(S+n R)$ that emits the four specific knots $K_{n}=N([(3 n-1) / 3])$ for $n=1,2,3,4$ must be of the form $S=1 /[-3]$ and $R=[1]$. It was in this way (with a finite number of observations) that the structure of recombination in $T_{n 3}$ resolvase was determined $[35]$.

In this version of the tangle model for DNA recombination we have made a blanket assumption that the substrate tangle $S$ and the recombination tangle $R$ and all the tangles $S+n R$ were rational. Actually, if we assume that $S$ is rational and that $S+R$ is rational, then it follows that $R$ is an integer tangle. Thus $S$ and $R$ neccesarily form a DNA knitting machine under these conditions. It is relatively natural to assume that $S$ is rational on the grounds of simplicity. On the other hand it is not so obvious that the recombination tangle should be an integer. The fact that the products of the DNA recombination experiments yield rational knots and links, lends credence to the hypothesis of rational tangles and hence integral recombination tangles. But there certainly is a subtlety here, since we know that the numerator closure of the sum of two rational tangles is always a rational knot or link. In fact, it is here that some deeper topology shows that certain rational products from a generalized knitting machine of the form $K_{n}=N(S+n R)$ where $S$ and $R$ are arbitrary tangles will force the rationality of the tangles $S+n R$. We refer the reader to $[\mathbf{1 0}],[\mathbf{1 1}],[\mathbf{8}]$ for the details of this approach.

Acknowledgments. It gives us great pleasure to thank John Conway, De Witt Sumners and Ray Lickorish for useful conversations. Also, we thank the referee for helpful remarks. 


\section{References}

[1] C. Bankwitz and H.G. Schumann, Über Viergeflechte, Abh. Math. Sem. Univ. Hamburg, 10 (1934), 263-284.

[2] S. Bleiler and J. Moriah, Heegaard splittings and branched coverings of $B^{3}$, Math. Ann., 281, 531-543.

[3] G. Burde, Verschlingungsinvarianten von Knoten und Verkettungen mit zwei Brücken, Math. Zeitschrift, 145 (1975), 235-242.

[4] G. Burde, H. Zieschang, "Knots", de Gruyter Studies in Mathematics 5 (1985).

[5] J.H. Conway, An enumeration of knots and links and some of their algebraic properties, Proceedings of the conference on Computational problems in Abstract Algebra held at Oxford in 1967, J. Leech ed., (First edition 1970), Pergamon Press, 329-358.

[6] N. Cozzarelli, F. Dean, T. Koller, M. A. Krasnow, S.J. Spengler and A. Stasiak, Determination of the absolute handedness of knots and catenanes of DNA, Nature, 304 (1983), 550-560.

[7] M.C. Culler, C.M. Gordon, J. Luecke and P.B. Shalen, Dehn surgery on knots, Annals of Math., 125 (1987), 237-300.

[8] I. DARCY, Solving tangle equations using four-plats, to appear in J. Knot Theory and its Ramifications.

[9] C.Ernst, D.W. Sumners, The growth of the number of prime knots, Math. Proc. Camb. Phil. Soc., 102 (1987), 303-315.

[10] C.Ernst, D.W. Sumners, A calculus for rational tangles: Applications to DNA Recombination, Math. Proc. Camb. Phil. Soc., 108 (1990), 489-515.

[11] C. ERnst, D. W. Sumners, Solving tangle equations arising in a DNA recombination model. Math. Proc. Cambridge Philos. Soc., 126, No. 1 (1999), 23-36.

[12] J.R. Goldman, L.H. Kauffman, Knots, Tangles and Electrical Networks, Advances in Applied Math., 14 (1993), 267-306.

[13] J.R. Goldman, L.H. Kauffman, Rational Tangles, Advances in Applied Math., 18 (1997), 300-332.

[14] L.H. Kauffman, "On knots", Ann. of Math. Stud. 115, Princeton Univ. Press, Princeton, N.J. (1987).

[15] L.H. Kauffman, S. Lambropoulou, On the classification of rational tangles, submitted for publication. (See http://www.math.uic.edu/ ₹auffman/ or http://users.ntua.gr/sofial)

[16] L.H. Kauffman, S. LAmbropoulou, On the classification of rational knots, submitted for publication. (See http://www.math.uic.edu/^kauffman/ or http://users.ntua.gr/sofial)

[17] A. Kawauchi, "A survey of knot theory", Birkhäuser Verlag (1996).

[18] A.Ya. Khinchin, "Continued Fractions", Dover (1997) (republication of the 1964 edition of Chicago Univ. Press).

[19] K. Kolden, Continued fractions and linear substitutions, Archiv for Math. og Naturvidenskab, 6 (1949), 141-196.

[20] W.B.R. LicKORISH, "An introduction to knot theory", Springer Graduate Texts in Mathematics, 175 (1997).

[21] W. Menasco, M. Thistlethwaite, The classification of alternating links, Annals of Mathematics, 138 (1993), 113-171.

[22] J.M. Montesinos, Revetements ramifies des noeuds, Espaces fibres de Seifert et scindements de Heegaard, Publicaciones del Seminario Mathematico Garcia de Galdeano, Serie II, Seccion 3 (1984).

[23] K. Murasugi, "Knot theory and its applications", Translated from the 1993 japanese original by B. Kurpita, Birkhäuser Verlag (1996).

[24] C.D. Olds, "Continued Fractions", New Mathematical Library, Math. Assoc. of Amerika, 9 (1963).

[25] L. Person, M. Dunne, J. Deninno, B. Guntel and L. Smith, Colorings of rational, alternating knots and links, (preprint 2002).

[26] V.V. Prasolov, A.B. Sossinsky, "Knots, Links, Braids and 3-Manifolds", AMS Translations of Mathematical Monographs 154 (1997).

[27] K. Reidemeister, "Knotentheorie" (Reprint), Chelsea, New York (1948).

[28] K. Reidemeister, Knoten und Verkettungen, Math. Zeitschrift, 29 (1929), 713-729.

[29] K. Reidemeister, Homotopieringe und Linsenräume, Abh. Math. Sem. Hansischen Univ., 11 (1936), 102-109. 
[30] D. Rolfsen, "Knots and Links", Publish or Perish Press, Berkeley (1976).

[31] H. Seifert, Die Verschlingungsinvarianten der zyklischen Knotenüberlagerungen, Abh. Math. Sem. Univ. Hamburg, 11 (1936), 84-101.

[32] J. SAWOLLEK, Tait's flyping conjecture for 4-regular graphs, preprint (1998).

[33] H. Schubert, Knoten mit zwei Brücken, Math. Zeitschrift, 65 (1956), 133-170.

[34] L. Siebenmann, Lecture Notes on Rational Tangles, Orsay (1972) (unpublished).

[35] D.W. Sumners, Untangling DNA, Math.Intelligencer, 12 (1990), 71-80.

[36] C. Sundberg, M. Thistlethwaite, The rate of growth of the number of alternating links and tangles, Pacific J. Math., 182 No. 2 (1998), 329-358.

[37] P.G. Tait, On knots, I, II, III, Scientific Papers, 1 (1898), Cambridge University Press, Cambridge, 273-347.

[38] H.S. WALL, "Analytic Theory of Continued Fractions", D. Van Nostrand Company, Inc. (1948).

Department of Mathematics, Statistics and Computer Science, University of Illinois at Chicago, 851 South Morgan St., Chicago IL 60607-7045, U.S.A.

E-mail address: kauffman@math.uic.edu

National Technical University of Athens, Department of Mathematics, Zografou Campus, GR-157 80 Athens, Greece.

E-mail address: sofia@math.ntua.gr 